\title{
Network analysis of membranous glomerulonephritis based on metabolomics data
}

\author{
AMIR TAHERKHANI ${ }^{1}$, SHIVA KALANTARI $^{2}$, AFSANEH AREFI OSKOUIE $^{3}$, \\ MOHSEN NAFAR ${ }^{4}$, MOHAMMAD TAGHIZADEH $^{5}$ and KOOROSH TABAR ${ }^{6}$
}

\author{
${ }^{1}$ Proteomics Research Center, Faculty of Paramedical Sciences, Shahid Beheshti University of Medical Sciences, \\ Tehran 1971653313; ${ }^{2}$ Chronic Kidney Disease Research Center, Shahid Labbafinejad Hospital, Shahid Beheshti \\ University of Medical Sciences, Tehran 1666663111; ${ }^{3}$ Department of Basic Science, Faculty of Paramedical Sciences, \\ Shahid Beheshti University of Medical Sciences, Tehran 1971653313; ${ }^{4}$ Urology Nephrology Research Center, \\ Shahid Labbafinejad Hospital, Shahid Beheshti University of Medical Sciences, Tehran 1666663111; \\ ${ }^{5}$ Bioinformatics Department, Institute of Biochemistry and Biophysics, Tehran University, Tehran 1417614411; \\ ${ }^{6}$ Chemistry and Chemical Engineering Research Center of Iran, Tehran 1496813151, Iran
}

Received November 17, 2017; Accepted June 29, 2018

DOI: $10.3892 / \mathrm{mmr} .2018 .9477$

\begin{abstract}
Membranous glomerulonephritis (MGN) is one of the most frequent causes of nephrotic syndrome in adults. It is characterized by the thickening of the glomerular basement membrane in the renal tissue. The current diagnosis of MGN is based on renal biopsy and the detection of antibodies to the few podocyte antigens. Due to the limitations of the current diagnostic methods, including invasiveness and the lack of sensitivity of the current biomarkers, there is a requirement to identify more applicable biomarkers. The present study aimed to identify diagnostic metabolites that are involved in the development of the disease using topological features in the component-reaction-enzyme-gene (CREG) network for MGN. Significant differential metabolites in MGN compared with healthy controls were identified using proton nuclear magnetic resonance and gas chromatography-mass spectrometry techniques, and multivariate analysis. The CREG network for MGN was constructed, and metabolites with a high centrality and a striking fold-change in patients, compared with healthy controls, were introduced as putative diagnostic biomarkers. In addition, a protein-protein interaction (PPI) network, which was based on proteins associated with MGN, was built and analyzed using PPI analysis methods, including molecular complex detection and ClueGene Ontology. A total of 26 metabolites were identified
\end{abstract}

Correspondence to: Dr Afsaneh Arefi Oskouie, Department of Basic Science, Faculty of Paramedical Sciences, Shahid Beheshti University of Medical Sciences, Darband Street, Tehran 1971653313, Iran

E-mail: a.arefi@sbmu.ac.ir

Key words: membranous glomerulonephritis, metabolite, network, biomarker, protein-protein interaction as hub nodes in the CREG network, 13 of which had salient centrality and fold-changes: Dopamine, carnosine, fumarate, nicotinamide D-ribonucleotide, adenosine monophosphate, pyridoxal, deoxyguanosine triphosphate, L-citrulline, nicotinamide, phenylalanine, deoxyuridine, tryptamine and succinate. A total of 13 subnetworks were identified using PPI analysis. In total, two of the clusters contained seed proteins (phenylalanine-4-hydroxlylase and cystathionine $\gamma$-lyase) that were associated with MGN based on the CREG network. The following biological processes associated with MGN were identified using gene ontology analysis: 'Pyrimidine-containing compound biosynthetic process', 'purine ribonucleoside metabolic process', 'nucleoside catabolic process', 'ribonucleoside metabolic process' and 'aromatic amino acid family metabolic process'. The results of the present study may be helpful in the diagnostic and therapeutic procedures of MGN. However, validation is required in the future.

\section{Introduction}

Membranous glomerulonephritis (MGN) is the most common cause of nephrotic syndrome in adults $>60$ years old $(1,2)$. Rivera et al (3) suggested that MGN was the pathology in 15.9-32.9\% of all nephrotic syndrome cases. Of all cases, $75 \%$ are classified as primary or idiopathic MGN; secondary MGN is associated with various conditions, including hepatitis infection, malignancy, systematic lupus erythematosus and drug intoxication $(4,5)$. MGN is histopathologically characterized by the thickening of the glomerular basement membrane (GBM) and the subepithelial deposition of immune complexes (5). Electron microscopy has demonstrated that subepithelial/intramembranous immune deposits (immunoglobulin $\mathrm{G}$ and complement) cause podocyte damage, which is usually associated with nephrotic syndrome (4). A deterioration in renal function and the development of end-stage renal disease occur in $\sim 40 \%$ of patients with primary MGN (5). Proteinuria $(6,7)$, edema $(8,9)$, hyperlipidemia $(10,11)$ and 
hypoalbuminemia $(12,13)$ are non-specific clinical symptoms of MGN. However, renal biopsy is the only definitive diagnostic approach, although it is an invasive procedure. Despite research in this field, the exact mechanisms of the development and progression of the disease have not been fully elucidated (14).

Systems biology gives meaning to large amounts of data derived from '-omics-' technologies (15). The '-omics-' technologies refer to high-throughput techniques, which may simultaneously detect a large number of molecules (including genes, transcriptomes, proteins and metabolites) in complex bio-samples (16). This approach may reduce the restrictions in the field of diagnosis and contribute to the understanding of the mechanisms involved in the pathogenesis of disease.

Metabolites are downstream products of gene expression and protein activity; therefore, they may provide an immediate indication of the biological phenotype (17). Nuclear magnetic resonance (NMR) (18) and mass spectrometry (MS) (19) are two techniques generally used to determine features of metabolomes. NMR has an outstanding reproducibility and quantitative accuracy, and may detect structures of unknown metabolites (18). Additionally, metabolite profiles captured by NMR are approximately independent of the operator, which provides high levels of reliability to the obtained results (17), Whereas, MS analyses lipids, which have low resolution in NMR. Novel mass spectrometers provide mass accuracy of compounds extending into the sub-5 parts per million regime, which makes determining the molecular formula possible (20). Therefore, the combination of NMR and MS technologies provides a more reliable biomarker discovery and makes the metabolomics field more challenging (17).

A considerable number of metabolites have previously been detected and introduced as potential biomarkers of various diseases (20). Sui et al (21) studied the metabolic profile of serum in three groups; healthy controls, patients with grade I-II immunoglobulin A nephropathy (IgA.N) and grade IV-V IgA.N. Sui et al (21) identified 21 metabolites with significant dysregulated expression in patients with IgA.N compared with healthy controls. In addition, they constructed an orthogonal partial least squares discriminant analysis (OPLS-DA) model to classify IgA.N from healthy controls. Their results demonstrated considerable $\mathrm{R}^{2}\left(\mathrm{R}^{2} \mathrm{X}=28.5 \% ; \mathrm{R}^{2} \mathrm{Y}=0.836\right)$ and $\mathrm{Q}^{2}(0.724)$ values with high sensitivity $(88.6 \%)$ and specificity (97.1\%) for diagnosing patients with $\operatorname{Ig}$ A.N.

It is noteworthy that the metabolome is more closely associated with phenotype, compared with the genome, transcriptome and proteome (22). Therefore, the present study aimed to investigate the metabolic differences between MGN and healthy controls using proton $\left({ }^{1} \mathrm{H}\right) \mathrm{NMR}$ and gas chromatography-mass spectrometry (GC-MS) techniques. Additionally, a component-reaction-enzyme-gene (CREG) network was constructed to determine more effective metabolites associated with the disease. Furthermore, a protein-protein interaction (PPI) network was assembled to identify biological processes (BPs) involved in the pathogenesis of MGN. However, genetic-based approaches (including reverse transcription-polymerase chain reactions) are necessary for biomarker validation.

In the present study, patients with MGN had significant differential metabolites compared with healthy controls, as demonstrated using ${ }^{1} \mathrm{H}$ NMR and GC-MS metabolomics approaches and advanced multivariate statistical analysis. Subsequently, the association of the metabolites with genes, enzymes and reactions that were associated with the disease, was investigated using network analyses.

\section{Materials and methods}

Sample collection and preparation. Urine samples $(450 \mu \mathrm{l})$ from patients with biopsy-proven MGN $(n=31)$ and normal volunteers $(n=21)$ were collected for the ${ }^{1} \mathrm{H}$ NMR experiments. Another cohort composed of 32 and 30 urine samples $(260 \mu \mathrm{l})$ from MGN and normal controls, respectively, was used for the GC-MS analyses. It was not possible to use the same cohorts for the two different techniques in the present study. Notably, Nobakht et al (23) additionally used two different cohorts in their previous study to identify novel biomarkers in the serum of sulfur mustard-exposed individuals (SMEIs) compared with healthy controls. Nobakht et al (23) collected a total of 29 serum samples, which included 12 healthy controls and 17 SMEIs for NMR analysis; six healthy controls and eight SMEIs were randomly selected for the GC-MS experiments (23). In the present study, 35 samples were common for ${ }^{1} \mathrm{H}$ NMR and GC-MS sections. As $>50 \%$ of the samples were the same, the present results are reliable; however, the results would be more significant if the same sample groups had been used. The demographic characteristics of the patients with MGN and the normal volunteers are presented in Table I. All urine samples were collected from patients at Labbafinejad Hospital (Tehran, Iran) between March 2015 and July 2016. To prepare the samples for analysis using ${ }^{1} \mathrm{H}$ NMR and GC-MS, $\mathrm{NaN}_{3}(10 \mathrm{mM})$ was first added to the samples. The samples were centrifuged $\left(3,220 \mathrm{x}\right.$ g for $20 \mathrm{~min}$ at $\left.4^{\circ} \mathrm{C}\right)$ and the supernatant was stored in aliquots at $-80^{\circ} \mathrm{C}$. Signed informed consent was obtained from all patients and normal volunteers. All patients with any systematic diseases, diabetes mellitus, cancer or secondary MGN were excluded. The present study was approved by the Ethics Committee of Shahid Beheshti University of Medical Sciences (Tehran, Iran).

${ }^{1} \mathrm{H} N M R$ analysis. To prepare the samples for ${ }^{1} \mathrm{H}$ NMR analysis, 3-trimethylsilyl-2H4-propionic acid and deuterium oxide (99.9\% D; Sigma-Aldrich; Merck KGaA, Darmstadt, Germany) in phosphate buffer were added to each sample. The Bruker DRX-500 NMR spectrometer (Bruker Corporation, Ettlingen, Germany) was used, operating at $500.13 \mathrm{MHz}{ }^{1} \mathrm{H}$ resonance frequency at $298 \mathrm{~K}\left(25^{\circ} \mathrm{C}\right)$. The Carr-Purcell-Meiboom-Gill pulse sequence was applied to decrease the intensity of the spectra of the macromolecules $(24,25)$. ProMetab software (version Prometab_v3_3) in MATLAB (version 8.4; MathWorks, Cambridge, UK) was used to perform ${ }^{1} \mathrm{H}$ NMR spectrum partitioning, data normalizing and the shift alignment of the urine samples (26-29).

GC-MS analysis. For the GC-MS analysis, $50 \mu 15 \mathrm{mg} / \mathrm{ml}$ solution of urease (Sigma-Aldrich; Merck KGaA; cat. no. U4002) was added to the urine samples to reduce the concentration of the urea. Derivatization of the samples followed protein precipitation using acetonitrile and drying. The protocol was as follows: $740 \mu 1$ 99.9\% acetonitrile (Sigma-Aldrich; Merck $\mathrm{KGaA}$; cat. no. 100030) was added to urine samples $(260 \mu \mathrm{l})$ 
Table I. Demographic characteristics of normal volunteers and patients with MGN.

\begin{tabular}{lcc}
\hline Demographic characteristics & NMR & GC-MS \\
\hline Average age in normal volunteers, years & 40.86 & 38.6 \\
Average age in patients with MGN, years & 39.16 & 41.55 \\
Female/male ratio in normal control & $11 / 10$ & $19 / 11$ \\
volunteers & & \\
Female/male ratio in patients with MGN & $17 / 14$ & $12 / 20$ \\
\hline
\end{tabular}

MGN, membranous glomerulonephritis; nuclear magnetic resonance; GC-MS, gas chromatography-mass spectrometry.

and left at room temperature for $15 \mathrm{~min}$, and precipitated proteins were removed by centrifugation at 3,000 $\mathrm{x} g$ for $10 \mathrm{~min}$ at $4^{\circ} \mathrm{C}$. The supernatants $(750 \mu \mathrm{l})$ were transferred to microtubes and placed inside the Eppendorf Vacufuge Concentrator 5301 (Eppendorf, Hamburg, Germany) for $9 \mathrm{~h}$ to evaporate. Acetonitrile $(100 \mu \mathrm{l})$ was added to each microtube to dissolve the dried metabolites, and transferred to glass autosampler vials prior to derivatization. For this, $20 \mu \mathrm{l}$ methoxyamine hydrochloride (Sigma-Aldrich; Merck KGaA; cat. no. 226904; $40 \mathrm{mg} / \mathrm{ml}$ in pyridine) was added to samples and left at room temperature for $60 \mathrm{~min}$, followed by incubation at $37^{\circ} \mathrm{C}$ for $20 \mathrm{~min}$. Derivatization of metabolites was completed by adding $150 \mu \mathrm{l}$, O-Bis(trimethylsilyl)trifluoroacetamide with $1 \%$ trimethylchlorosilane (Sigma-Aldrich; Merck KGaA; cat. no. 33155-U). The spectra were obtained using an Agilent 6890 GC coupled to an Agilent 5973 inert EI/CI mass selective detector (Agilent Technologies, Inc., Santa Clara, CA, USA); the non-polar HP-1 capillary column ( $30 \mathrm{mx} 0.25 \mathrm{~mm}$ inner diameter; Agilent J\&W capillary column; Agilent Technologies, Inc.) was coated with $100 \%$ dimethylpolysiloxane with a film thickness of $0.5 \mu \mathrm{m}$. The temperature gradient varied between 40 and $280^{\circ} \mathrm{C}$ in a solvent delay of $9.5 \mathrm{~min}$ and a gas flow of $1 \mathrm{ml} / \mathrm{min}$. R software (version 3.3.3; R Core Team; www.r-project.org) and MATLAB (version 8.4) were used for data preprocessing, which included the baseline correction and shift alignment steps. Metabolite identification was performed using Enhanced ChemStation (version D.00.00.38; Agilent Technologies, Inc.) and Automated Mass Spectral Deconvolution and Identification System software (version 2.0d; National Institute of Standards and Technology, Gaithersburg, MD, USA). Only components with a matching factor of $\geq 70 \%$ were considered to be statistically significant.

Statistical analyses. Advanced supervised multivariate modeling, including OPLS-DA and partial least squares discriminant analysis (PLS-DA), were performed using R (version 3.3.3) and the XLSTAT (Addinsoft, New York, NY, USA) package, respectively. Principal component analysis was applied prior to OPLS-DA modeling for outlier identification of the GC-MS dataset, while PLS-DA was used for the NMR dataset. The variables with a variable importance in projection (VIP) score $>1$ were considered to be statistically significant. A receiver operating characteristic (ROC) curve was generated to evaluate the diagnostic ability of the PLS-DA model.
CREG network. A list of 70 differential metabolites that was derived from the experimental part of the study was used as the input data for the Cytoscape tool (3.4.0; www.cytoscape. org). The MetScape plugin (version 3.1.3; http://apps.cytoscape. org/apps/metscape) (30) was used to construct the CREG network. MetScape uses data from the Human Metabolome Database, the Kyoto Encyclopedia of Genes and Genomes (KEGG), and the Edinburgh Human Metabolic Network to determine the connections among the metabolites, genes, and enzymes (30-33).

The initial network was not fully connected and, therefore, the isolated components in the network were expanded using the MetScape expand function to access the fully connected CREG network with 1,148 nodes. To compute the centrality of all the components in the network, CentiScape 2.2 was used (34). To identify the most effective metabolites in the network, the 'Centroid unDir' and 'Betweenness unDir' of the nodes were calculated. The metabolites that had a centroid and betweenness greater than or equal to the mean of the nodes in the network were considered as hubs.

PPInetwork. The PPI network is an effective tool for systematic research into complex activities in cells (35). PPI refers to the physical or functional binding of two or more proteins (36). In the present study, a set of proteins associated with MGN was acquired from the Search Tool for the Retrieval of Interacting Genes database (string-db.org), which was based on selected proteins from the CREG network (37). Cytoscape 3.4.0 was used to visualize the PPI network. Subsequently, the clustering algorithm 'molecular complex detection' (MCODE) was used to identify highly dense combined regions in the PPI network. MCODE is a plugin widely used in Cytoscape software that is used to find subnetworks in biological networks; this is performed based on vertex weighting by the neighborhood density and external traversal from a locally dense seed protein, the aim of which is to identify dense parts (38). In addition, MCODE may be run in a direct mode in which a seed node is identified as a vertex of the cluster, according to the existing biological knowledge of the system (39). The MCODE advanced options were as follows: Minimum score for clusters, 2; number of nodes involved in each cluster, $\geq 2$; and node score cutoff, 0.2 .

The BP categories of the most important clusters associated with MGN were analyzed using ClueGO (v2.3.3; Laboratory of Integrative Cancer Immunology, Paris, France). ClueGO is a plugin used in Cytoscape that combines gene ontology (GO) terms (including KEGG and BioCarta pathways) to make a functionally organized GO and/or pathway term network (40). In order to correct the P-values for multiple tests, statistical standard correction methods were imposed, including Bonferroni, Bonferroni step-down and Benjamini-Hochberg (40). Categories with $\mathrm{P} \leq 0.05$ were considered to indicate a statistically significant difference.

\section{Results}

Identification of differential metabolites using ${ }^{1} H$ NMR. A predictive model was built using PLS-DA for the ${ }^{1} \mathrm{H}$ NMR dataset. The model was able to distinguish MGN from normal controls with $91.49 \%$ sensitivity and $71.43 \%$ specificity. The 
quality parameters of the model were as follows: $R^{2} X=0.353$, $\mathrm{R}^{2} \mathrm{Y}=0.316$, and $\mathrm{Q}^{2}=0.275$ (Fig. 1). The $R O C$ curve of the model is demonstrated in Fig. 2. The area under the curve of the model was 0.889. A total of 63 differential metabolites with a VIP $>1$ were identified for MGN compared with the normal samples from the PLS-DA diagnostic model. All of the upregulated and downregulated differential metabolites in MGN are listed in Tables II and III, respectively.

Identification of differential metabolites using GC-MS. Following preprocessing, the normalized dataset consisted of 62 observations (MGN, 32; normal, 30) and 2,550 variables (peak numbers). This dataset was used to build the OPLS-DA diagnostic model. The parameters of the model were $\mathrm{R}^{2} \mathrm{X}=0.852, \mathrm{R}^{2} \mathrm{Y}=0.696$, and $\mathrm{Q}^{2}=0.614$, suggesting that the OPLS-DA model was statistically robust and able to strongly differentiate normal controls from patients with MGN (Fig. 3). Finally, seven metabolites were determined as statistically discriminating variables $(\mathrm{P} \leq 0.001)$ for patients with MGN compared with healthy individuals. All metabolites had a match factor $\geq 70 \%$ (Table IV).

CREG network analysis. The list of 70 differential metabolites derived using ${ }^{1} \mathrm{H}$ NMR and GC-MS was used as input data in MetScape 3.1.3; a total of 54 components were identified in the MetScape database. The fully connected CREG network, which was based on the pathways, consisted of 268 components, 251 biochemical reactions, 182 enzymes and 437 genes (Fig. 4). The CentiScape plugin was used to analyze the centrality of the component nodes. A total of 26 input metabolites had above average Betweenness unDir and Centroid unDir; accordingly, they were assigned as hub nodes in the CREG network (Table V). The Betweenness and Centroid diagrams of four hub metabolites (dopamine, nicotinamide D-ribonucleotide, nicotinamide and phenylalanine) are demonstrated in Fig. 5.

PPI analysis. The PPI network, which was based on the proteins derived from the CREG network and associated with MGN, contained 306 nodes and 6,088 edges (Fig. 6). The edges demonstrate either a physical or functional connection between two proteins. The network analysis by MCODE defined 13 clusters in the complex. Each cluster consisted of highly joined proteins that were presumably involved in a common BP (36). The characteristics of all clusters are illustrated in Table VI. The GO analysis was performed for two subnetworks (cluster 4 and 9) that had seed nodes involved in the initial list. Figs. 7 and 8 represent clusters 4 and 9, respectively. A total of 13 of the differential metabolites were considered to be potential biomarkers for MGN, according to their centralities and fold-changes (Table VII).

Identification of BPs affected in MGN. A total of five BPs were significantly affected in MGN. 'Pyrimidine-containing compound biosynthetic process', 'purine ribonucleoside metabolic process', 'nucleoside catabolic process' and 'ribonucleoside metabolic process' were associated with the protein family of cluster 4 (Fig. 9). 'Aromatic amino acid family metabolic process' was associated with the proteins involved in cluster 9 (Fig. 10).
Table II. A total of 30 metabolites were upregulated in patients with membranous glomerulonephritis compared with normal controls, identified by proton nuclear magnetic resonance.

\begin{tabular}{lccl}
$\begin{array}{l}\text { Chemical } \\
\text { shift, ppm }\end{array}$ & Fold-change & KEGG ID & \multicolumn{1}{c}{$\begin{array}{c}\text { Metabolite } \\
\text { name }\end{array}$} \\
\hline 6.62 & 1.36 & C02372 & 4-aminophenol \\
6.34 & 1.78 & C00364 & 5-thymidylic acid \\
8.30 & 1.43 & C00212 & Adenosine \\
5.34 & 1.96 & C00499 & Allantoic acid \\
6.14 & 2.04 & C00020 & AMP \\
7.10 & 1.67 & C00386 & Carnosine \\
6.58 & 1.42 & C00417 & Cis-aconitic acid \\
5.50 & 1.65 & C02226 & Citraconic acid \\
3.10 & 1.48 & C00097 & Cysteine \\
6.06 & 1.83 & C00475 & Cytidine \\
6.26 & 1.92 & C00881 & Deoxycytidine \\
5.30 & 2.00 & C00984 & D-galactose \\
6.30 & 1.84 & C00286 & dGTP \\
6.74 & 1.65 & C03758 & Dopamine \\
6.50 & 1.55 & C00122 & Fumaric acid \\
5.46 & 1.70 & C00329 & Glucosamine \\
4.14 & 1.35 & C00258 & Glyceric acid \\
6.66 & 1.38 & C00544 & Homogentisic acid \\
6.10 & 1.88 & C00294 & Inosine \\
7.06 & 1.47 & C00135 & L-histidine \\
6.78 & 1.55 & C00328 & L-kynurenine \\
6.90 & 1.30 & C00082 & L-tyrosine \\
6.02 & 1.63 & C01384 & Maleic acid \\
6.46 & 1.60 & C01732 & Mesaconic acid \\
6.22 & 2.01 & C00455 & Nicotinamide ribotide \\
6.18 & 1.99 & C00295 & Orotic acid \\
5.26 & 2.21 & C00250 & Pyridoxal \\
6.98 & 1.35 & C02341 & Trans-aconitic acid \\
6.94 & 1.34 & C00483 & Tyramine \\
6.38 & 1.69 & C00785 & Urocanic acid \\
& & & \\
\hline & & & \\
60 & & &
\end{tabular}

AMP, adenosine monophosphate; GTP, guanine triphosphate; KEGG, Kyoto Encyclopedia of Genes and Genomes.

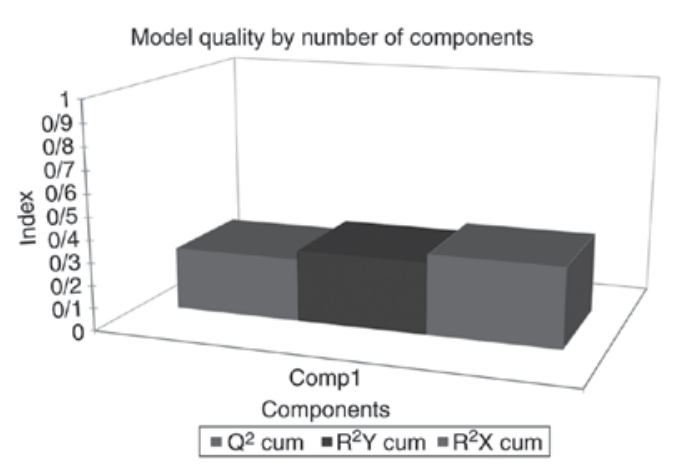

Figure 1. Partial least squares discriminant analysis differentiation model quality factors obtained from the proton nuclear magnetic resonance data of the urine samples. The three graphs on the $\mathrm{X}$-axis from the left to the right represent $Q^{2}, R^{2} Y$ and $R^{2} X$, respectively. The $y$-axis represents the score of each quality factor. Cum, cumulative. 
Table III. A total of 33 metabolites were downregulated in membranous glomerulonephritis patients compared with normal controls, identified by proton nuclear magnetic resonance.

\begin{tabular}{|c|c|c|c|}
\hline Chemical shift, ppm & Fold change & KEGG ID & Metabolite name \\
\hline 2.02 & 0.73 & C00986 & 1,3-diaminopropane \\
\hline 7.66 & 0.44 & $\mathrm{C} 01152$ & 1-methylhistidine \\
\hline 0.86 & 0.40 & C05984 & 2-hydroxybutyric acid \\
\hline 1.10 & 0.60 & C00109 & 2-ketobutyric acid \\
\hline 0.62 & 0.23 & C17727 & 3a,6a,7b-trihydroxy-5b-cholanoic acid \\
\hline 0.74 & 0.30 & $\mathrm{C} 03238$ & 5alpha-cholestanone \\
\hline 1.46 & 0.67 & $\mathrm{C} 00041$ & Alanine \\
\hline 2.70 & 0.52 & $\mathrm{C} 01262$ & Anserine \\
\hline 1.90 & 0.74 & $\mathrm{C} 00062$ & Arginine \\
\hline 1.30 & 0.60 & $\mathrm{C} 01585$ & Caproic acid \\
\hline 0.66 & 0.25 & C00695 & Cholic acid \\
\hline 2.54 & 0.69 & $\mathrm{C} 00158$ & Citric acid \\
\hline 1.58 & 0.64 & $\mathrm{C} 00327$ & Citrulline \\
\hline 4.06 & 0.67 & C00791 & Creatinine \\
\hline 7.86 & 0.38 & C00526 & Deoxyuridine \\
\hline 2.14 & 0.70 & C00064 & Glutamine \\
\hline 0.70 & 0.30 & C01921 & Glycocholic acid \\
\hline 3.82 & 0.74 & C00581 & Guanidoacetic acid \\
\hline 2.62 & 0.62 & $\mathrm{C} 00155$ & Homocysteine \\
\hline 1.14 & 0.43 & $\mathrm{C} 02632$ & Isobutyric acid \\
\hline 1.42 & 0.70 & C00186 & L-lactic acid \\
\hline 0.94 & 0.69 & $\mathrm{C} 00123$ & L-leucine \\
\hline 7.38 & 0.55 & C00079 & L-phenylalanine \\
\hline 2.38 & 0.54 & C00711 & Malic acid \\
\hline 1.26 & 0.57 & $\mathrm{C} 02170$ & Methylmalonic acid \\
\hline 7.58 & 0.36 & $\mathrm{C} 00153$ & Niacinamide \\
\hline 1.98 & 0.71 & C00148 & Proline \\
\hline 2.46 & 0.80 & $\mathrm{C} 00022$ & Pyruvic acid \\
\hline 1.82 & 0.71 & $\mathrm{C} 00750$ & Spermine \\
\hline 2.58 & 0.61 & C00042 & Succinic acid \\
\hline 0.58 & 0.25 & $\mathrm{C} 05122$ & Taurocholic acid \\
\hline 7.30 & 0.57 & C00398 & Tryptamine \\
\hline 7.70 & 0.67 & $\mathrm{C} 00385$ & Xanthine \\
\hline
\end{tabular}

KEGG, Kyoto Encyclopedia of Genes and Genomes.

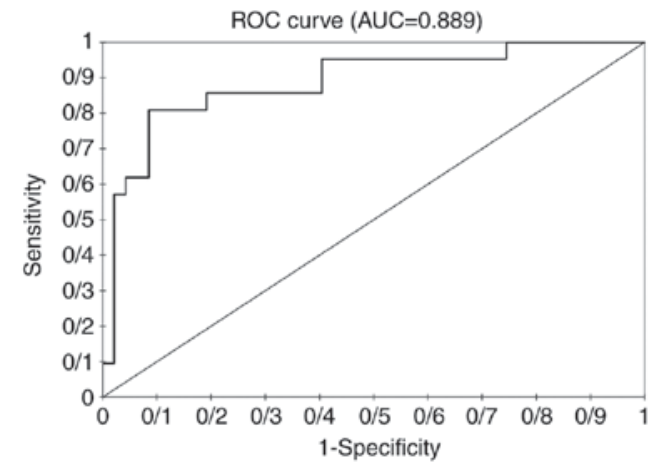

Figure 2. ROC analysis was performed to evaluate the classification model established with the partial least squares discriminant analysis algorithm. The AUC was 0.889. ROC, receiver operating characteristic; AUC, area under the curve.

\section{Discussion}

In the present study, the urinary metabolites from patients with MGN and healthy volunteers were analyzed using ${ }^{1} \mathrm{H}-\mathrm{NMR}$ and GC-MS techniques. A total of 70 differential metabolites were demonstrated to be statistically significant in the two groups. To the best of our knowledge, the present study is the first in which a CREG network associated with MGN was constructed and analyzed using the MetScape and CentiScape plugins in Cytoscape software. In addition, PPI analysis was performed to verify the BPs involved in the pathogenesis of MGN, using the MCODE and ClueGO plugins in the Cytoscape software.

Hallan et al (41) reported that non-diabetic patients with stages 3-4 chronic kidney disease (CKD) had reduced urinary 
Table IV. Seven differential urinary metabolites in patients with membranous glomerulonephritis compared with normal controls, identified by gas chromatography-mass spectrometry.

\section{A, Upregulated}

\begin{tabular}{llccc}
\hline Metabolite name & HMDB ID & KEGG ID & Match factor (\%) & Fold change, MGN/normal \\
\hline Octadecanoic acid & HMDB00827 & C01530 & 93 & 1.10 \\
Urea & HMDB00294 & C00086 & 92 & 1.09 \\
Palmitic acid & HMDB00220 & C00249 & 92 & 1.23 \\
Oleic acid & HMDB00207 & C00712 & 83 & 1.20 \\
\hline
\end{tabular}

B, Downregulated

\begin{tabular}{llccr}
\hline Metabolite name & HMDB ID & KEGG ID & Match factor (\%) & Fold change, MGN/normal \\
\hline N-acetyl-D-glucosamine & HMDB00215 & C00140 & 75 & 0.91 \\
Methylmalonic acid & HMDB00202 & C02170 & 72 & 0.05 \\
Glycocholic acid & HMDB00138 & C01921 & 70 & 0.88
\end{tabular}

KEGG, Kyoto Encyclopedia of Genes and Genomes; MGN, membranous glomerulonephritis.

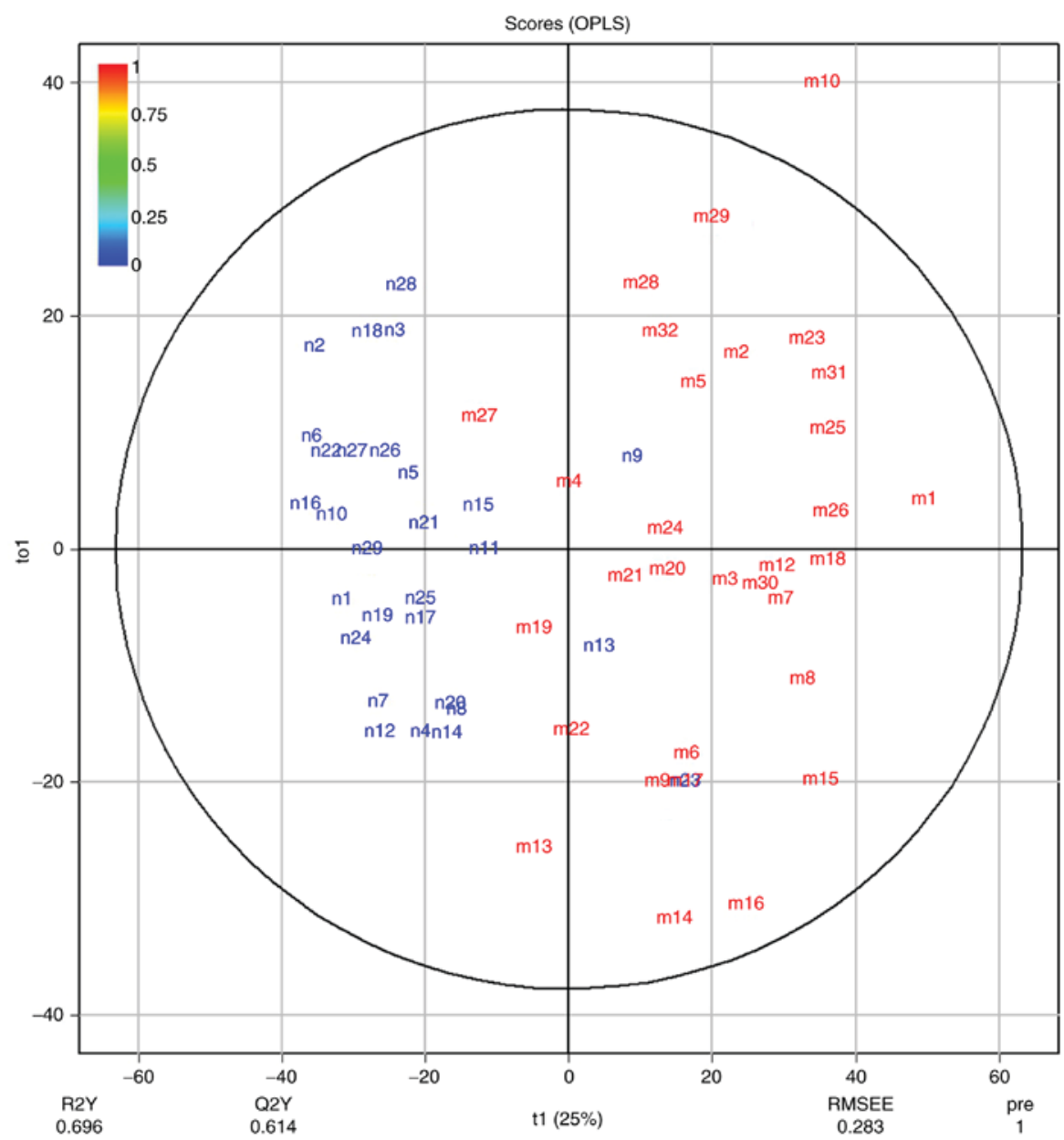

Figure 3. OPLS discriminant analysis score plots in the predictive (x-axis) and orthogonal (y-axis) components of the gas chromatography-mass spectrometry data of the urine samples. n, normal controls; m, patients with membranous glomerulonephritis; OPLS, orthogonal projections to latent structures.

excretion of succinate compared with healthy controls. These patients exhibited reduced mRNA expression levels of adenosine monophosphate (AMP)-activated protein kinase and peroxisome proliferator-activated receptor- $\gamma$ coactivator- $1 \alpha$, 


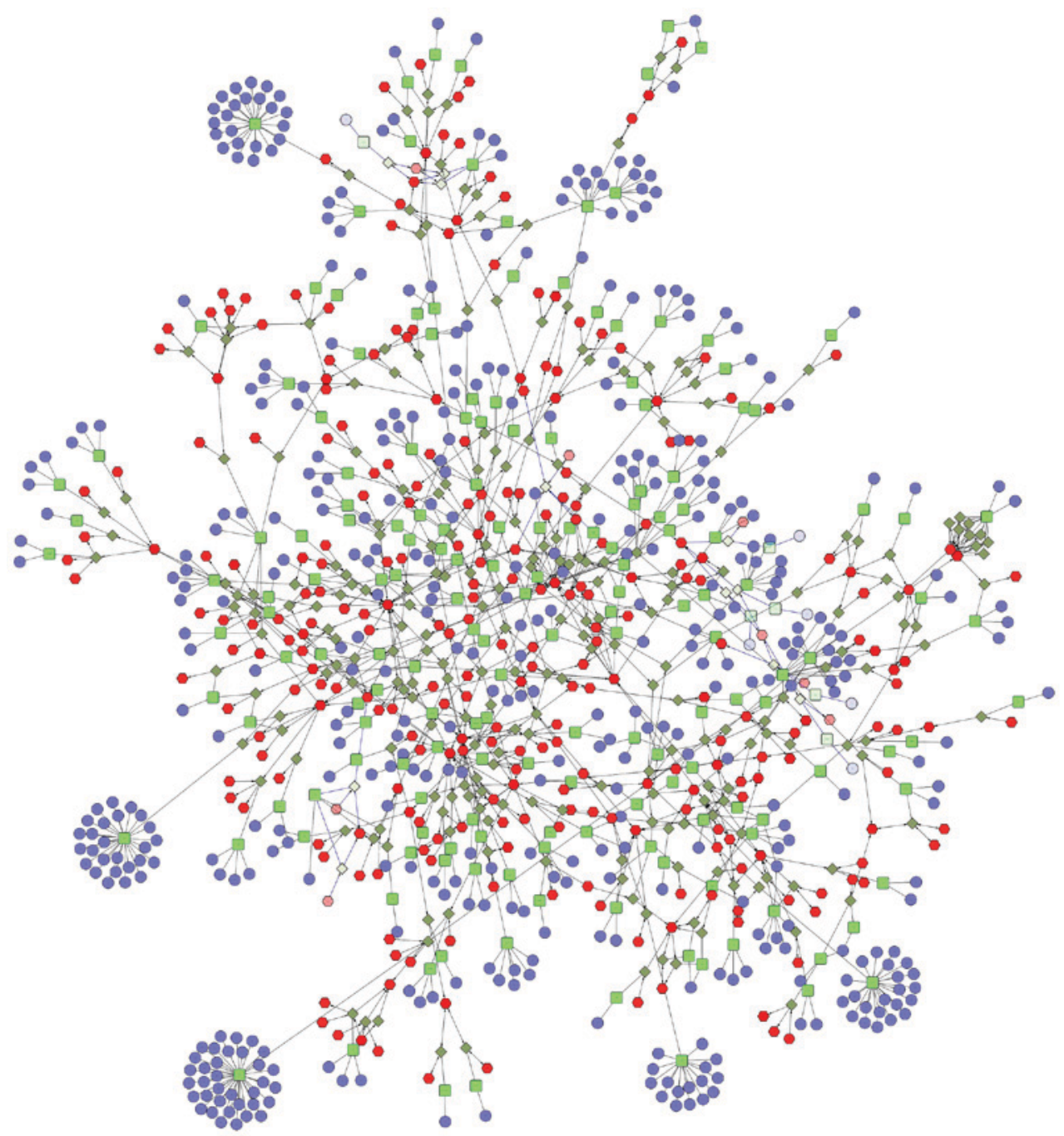

Figure 4. Fully connected component-reaction-enzyme-gene network. The violet circles represent genes, the green squares are enzymes, the dark green diamonds represent reactions and the hexagonal nodes indicate components; the red hexagons are the input metabolites.

which are the two key regulators of mitochondrial biogenesis in the renal tissue of non-diabetic patients with CKD. This previous study additionally reported a significant reduction in the expression of genes involved in succinate regulation, including mitochondrial succinyl-coenzyme A (succinyl-CoA) ligase (adenosine diphosphate-forming) subunit $\beta$, mitochondrial succinyl-CoA ligase (GDP-forming) subunit $\alpha$, and mitochondrial succinyl-CoA ligase [guanosine diphosphate (GDP)-forming) subunit $\beta$, in the glomeruli and tubulointerstitial compartments of the kidneys in non-diabetic patients with CKD. Therefore, the low urinary levels of succinate in patients with MGN may additionally be due to the decreased expression of the proteins that are associated with mitochondrial biogenesis. However, validation tests are required. In addition, Toyohara et al (42) identified a negative correlation between the plasma concentration of succinate and the estimated glomerular filtration rate (eGFR) in a wide range of patients with CKD, including patients with MGN, focal segmental glomerulosclerosis (FSGS), IgA.N and diabetic nephropathy (DN), compared with the levels in healthy controls. It was suggested that compounds that have a negative correlation with eGFR may be considered to be uremic toxins, and thus may be useful in detecting CKD.
Lin et al (43) studied 55 pediatric patients with mild-to-moderate CKD and demonstrated that $70 \%$ of the patients had at least one blood pressure load abnormality on ambulatory blood pressure monitoring. It was reported that a low urinary citrulline (CIT) level and CIT/arginine (ARG) ratio were associated with blood pressure load abnormalities in children with early CKD. It was suggested that in patients with mild-to-moderate CKD, the kidney experiences a compensatory increase in CIT uptake and conversion of CIT to ARG, to increase the renal ARG bioavailability in response to the enhanced blood pressure load. Since ARG is a substrate for nitric oxide (NO) synthase, which is used to generate NO and CIT, NO deficiency contributes to hypertension, cardiovascular disease and CKD. Therefore, it may be speculated that the decreased urinary levels of CIT in patients with MGN occur due to the increased uptake of CIT by the kidneys in order to convert the CIT to ARG and elevate the production of NO, although this requires confirmation.

Carnosine ( $\beta$-alanyl-L-histidine) is a natural reactive oxygen species (ROS) scavenger (44). Carnosine has renoprotective effects and may possibly be used to treat DN (45). Kaori et al (46) reported low urinary levels of carnosine 
in patients with stages 1-2 CKD compared with healthy controls. However, the researchers did not declare the causes of CKD. According to the present results, urinary carnosine was increased in patients with MGN compared with healthy controls. It may be speculated that the elevated level of urinary carnosine in patients with MGN is due to increased ROS levels in the renal tissue; therefore, the requirement for carnosine in the kidney is evident, and a large amount of carnosine is produced.

Pyridoxal 5'-phosphate (PLP) and pyridoxal (PL) are two types of vitamin B6. Prior to entering a cell, PLP is dephosphorylated to PL by the enzyme alkaline phosphatase; PL is subsequently converted to PLP by pyridoxine kinase (47). Since the reaction between PLP and PL is reversible and widely distributed, plasma levels of PLP + PL may be considered to be a single pool of vitamin B6 $(47,48)$. Chen et al $(48)$ demonstrated higher levels of serum PLP in patients with stages 4-5 CKD compared with patients with stages 2-3 CKD. The principal etiologies of the CKD were DN, IgA nephrology, gouty nephropathy, nephrotic syndrome and tubulointerstitial nephritis. The authors reported that vitamin B6 is an important factor in the inflammatory responses in patients with CKD and patients undergoing hemodialysis. Zhang et al (49) revealed that high physiological concentrations of PLP and PL prohibited the activation of Toll-like receptor-induced nuclear factor (NF)- $\gamma \mathrm{B}$ and NACHT, LRR and PYD domains-containing protein 3-mediated caspase-1 to inhibit the production of interleukin $1 \beta$ (IL-1 $\beta$ ) in macrophages. In addition, Chen et al (48) suggested that an enhanced vitamin B6 status is required for patients with CKD and patients undergoing hemodialysis to manage their inflammatory responses. Therefore, according to the present results, increased levels of urinary PL in patients with MGN may be due to the inflammatory responses of the renal tissue, although this requires confirmation.

Egashira et al (50) compared the alteration in tryptophan-niacin metabolism in rats with puromycin aminonucleoside (PAN)-induced nephrosis with that of a control group. It was demonstrated that the urinary excretion of nicotinamide and its metabolites was significantly lower in PAN-treated rats compared with control rats. It has been reported that a number of CKD-associated secretory phenotype proteins, including transforming growth factor- $\beta$ (TGF- $\beta$ ), are involved in the early and late stages of renal wound healing in CKD (51-53). TGF- $\beta 1$ appears to serve an important role in mediating the hypertrophic and fibrotic/sclerotic manifestations of DN (53). The protein sirtuin 1 (SIRT1; a class III histone deacetylase) is able to inhibit TGF- $\beta 1$-induced apoptosis in glomerular mesangial cells through the deacetylation of mothers against decapentaplegic homolog 7 (54). The upregulation of SIRT1 additionally reduces ROS-induced apoptosis in mesangial cells and may provide a novel method for treating glomerular diseases (55). Li et al (56) demonstrated that the decreased expression of SIRT1 in the kidneys of rats with DN, were restored following treatment with $2,3,5,4$ '-tetrahydroxystilbene-2-O- $\beta$-D-glucoside (TSG). It was additionally reported that nicotinamide, which is an inhibitor of SIRT1, partially attenuates the inhibitory effect of TSG on TGF- $\beta 1$ expression under high glucose conditions. According to the results of other studies, in addition to the present study, it may be speculated that the production of nicotinamide is not decreased in patients with MGN; rather, the excretion of
Table V. A total of 26 hub metabolites were identified in the component-reaction-enzyme-gene network associated with membranous glomerulonephritis.

\begin{tabular}{ll}
\hline Metabolite name & KEGG ID \\
\hline L-leucine & C00123 \\
Dopamine & C03758 \\
Tyramine & C00483 \\
Carnosine & C00386 \\
L-histidine & C00135 \\
L-citrulline & C00327 \\
Fumarate & C00122 \\
Xanthine & C00385 \\
L-glutamine & C00064 \\
Pyruvate & C00022 \\
L-alanine & C00041 \\
Nicotinamide D-ribonucleotide & C00455 \\
Adenosine & C00212 \\
AMP & C00020 \\
L-arginine & C00062 \\
Nicotinamide & C00153 \\
L-tyrosine & C00082 \\
L-phenylalanine & C00079 \\
L-cysteine & C00097 \\
L-proline & C00148 \\
Pyridoxal & C00250 \\
dGTP & C00286 \\
Deoxyuridine & C00526 \\
Tryptamine & C00398 \\
Succinate & C00042 \\
Citrate & C00158
\end{tabular}

AMP, adenosine monophosphate; GTP, guanine triphosphate; KEGG, Kyoto Encyclopedia of Genes and Genomes.

its metabolites may decrease. Therefore, it was hypothesized that in renal tissue of patients with MGN, the presence of nicotinamide inhibits SIRT1, increases the expression of TGF- $\beta 1$, enhances the levels of ROS and increases renal damage. Notably, Kalantari et al (57) demonstrated a decreased urinary excretion level of N-methylnicotinamide in patients with FSGS with proteinuria $>3,000 \mathrm{mg} /$ day, compared with patients with FSGS with proteinuria $<3,000 \mathrm{mg} /$ day; the authors proposed that a decreased level of nicotinamide mononucleotide (NMN) was an indication of tubular secretion dysfunction. Therefore, reduced urinary excretion of nicotinamide may additionally be due to tubular secretion dysfunction, although future experiments are required to verify the above.

The protein nicotinamide phosphoribosyltransferase (Nampt) is a rate-limiting enzyme in the nicotinamide adenine dinucleotide (NAD) salvage pathway, which converts nicotinamide to NMN in mammals to enable $\mathrm{NAD}^{+}$biosynthesis (58). Chen et al (59) demonstrated that the endogenous expression levels of Nampt and NF-kB p65 were significantly increased in HBZY-1 cells subjected to high glucose for different time 
A

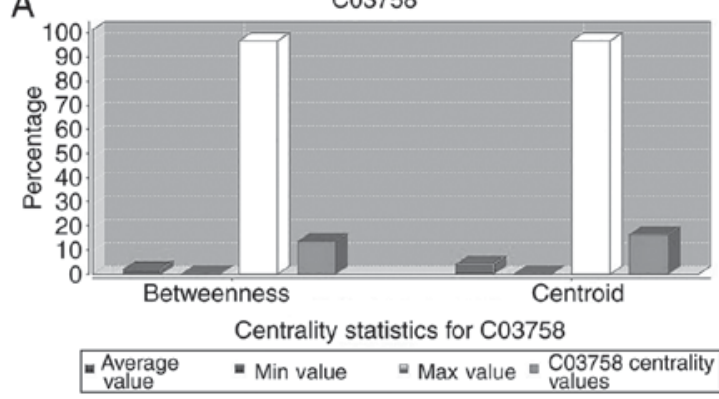

C

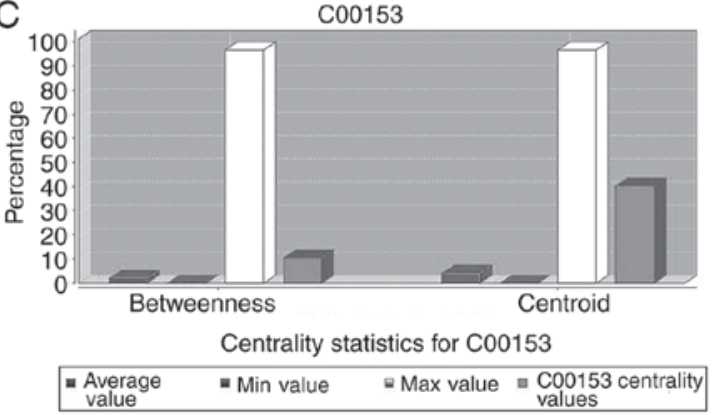

$\mathrm{B}$

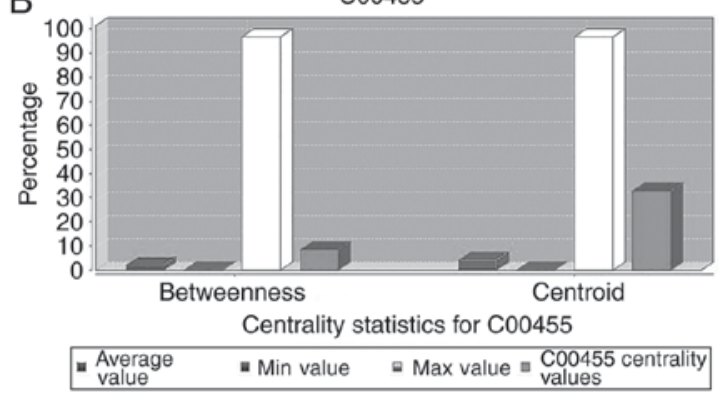

D

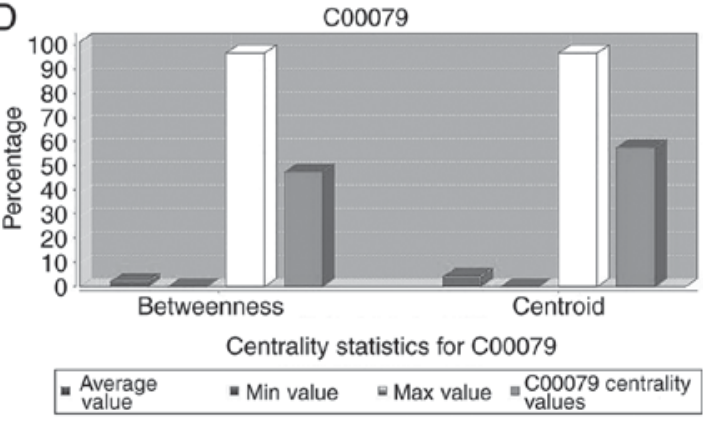

Figure 5. Betweenness and centroid diagrams. The betweenness and centroid diagrams of (A) dopamine, (B) nicotinamide D-ribonucleotide, (C) nicotinamide and (D) phenylalanine are presented. The $\mathrm{x}$-axis corresponds, from left to right, to the average value, minimum value, maximum value and metabolite centrality value, respectively. The y-axis represents the score of centrality in terms of percentage.

periods; whereas, the expression of SIRT1 was significantly downregulated. It was additionally demonstrated that the expression levels of Nampt and vimentin were significantly higher in an insulin gene-mutant diabetic C57/LB6 mouse model compared with the control group. The researchers suggested that there may be an association between Nampt and fibrotic factors, including vimentin and fibronectin. Furthermore, it was reported that NMN was able to activate SIRT1 by inhibiting the Nampt pathway, and that SIRT1 was upregulated in an NMN-treated group in vitro and in vivo compared with the untreated group. Therefore, the authors suggested that NMN may induce the downregulation of vimentin expression by interacting with $N F-\kappa B$ p65. It was additionally suggested that NMN may inhibit Nampt upregulation through negative feedback to indirectly inhibit NF- $\mathrm{B}$ p65-dependent inflammatory responses in HBZY-1 cells. Hasegawa et al (60) demonstrated that SIRT1 was downregulated in the proximal tubules (PT) prior to the occurrence of albuminuria in streptozotocin-induced or obese-type $(\mathrm{db} / \mathrm{db})$ diabetic mice. This downregulation decreased the excretion of NMN from the PT into the podocytes in the glomeruli. Hasegawa et al (60) demonstrated that decreased uptake of NMN in the podocytes results in a decrease in SIRT1 expression and an increase in the expression of the tight junction protein claudin- 1 in the podocytes. The enhanced expression of claudin-1 ultimately leads to more albuminuria in DN. Therefore, it was suggested that NMN has renoprotective effects, as its deficiency results in the increased expression of fibrotic/inflammatory factors in DN (60). According to the present results, there were increased expression levels of NMN in the urine of patients with MGN compared with the normal controls. Although no studies, to the best of our knowledge, have been performed on the role of NMN in the pathogenesis of MGN, it may be suggested that similar mechanisms may be involved in DN and MGN that prevent the excretion of NMN from the PT into the glomeruli, although this requires confirmation. Instead, NMN is released into the urine, which accounts for the high levels of the molecule observed.

AMP deaminase (Ampd) is the rate-limiting enzyme in the catabolism of AMP to uric acid and is a component of the purine nucleotide cycle, which is responsible for the deamination of AMP to inosine monophosphate (61). Helmering et al (61) reported that a mutation in the Ampd2 gene (a liver isoform of Ampd) leads to nephrotic syndrome and hypercholesterolemia in Ampd2 knockout (KO) mice. It was additionally observed that the Ampd2 $\mathrm{KO}$ mice had minimal alterations in the kidneys, minimal to moderate thickening of the GBM, an increase in the cellularity of the mesangial matrix and an increase in inflammatory cells in the glomeruli. In addition, the authors performed a metabolomics analysis on the liver tissue of KO mice using GC-MS and liquid chromatography-tandem mass spectrometry, and demonstrated a significant increase in hepatic AMP expression levels. They suggested that Ampd2 function was critical for podocyte survival. According to the present results, high expression levels of urinary AMP were observed in patients with MGN. Whether the activity or the expression of Ampd had been affected in the renal tissues of the patients with MGN was not confirmed in the present study; future validation studies may clarify these issues.

Significant increased urinary levels of deoxyguanine triphosphate (dGTP) in patients with MGN compared with healthy controls were detected. Kalantari et al (62) additionally reported a significant increased urinary excretion of dGTP in patients with severe stages of $\operatorname{IgA}$.N compared with patients with mild stages of IgA.N. The dGTP had a positive correlation with proteinuria in patients with IgA.N, which was determined using ${ }^{1} \mathrm{H}$ NMR spectroscopy. Furman et al (63) demonstrated increased levels of molecules of deoxyribonucleoside 
triphosphates, including dGTP and deoxyadenosine triphosphate, in Vero cells infected with herpes simplex virus type 1 treated with acyclovir, compared with untreated infected cells. They speculated that the increases were partly due to the inhibition of viral DNA polymerase activity, which resulted in the reduced utilization of the deoxyribonucleoside triphosphates. In addition, Gamboa et al (64) reported that diabetic and non-diabetic patients with stage 3-4 CKD had reduced mitochondrial DNA copy numbers. Accordingly, it may be suggested that the renal mitochondria are affected in patients with MGN, which results in reduced DNA synthesis in the organelle; therefore, a decrease in the consumption of dGTP leads to greater excretion of the molecule in the urine of patients, although this requires confirmation.

Low levels of urinary 2'-deoxyuridine (dU) in patients with MGN patients were detected. Although deoxycytidine (dC) was not considered to be a hub in the CREG network, a considerable fold-change in patients with MGN (1.92-fold increase compared with controls) was demonstrated. Xia et al (65) reported a significant increase in levels of cytosine and cytidine in the serum of patients with DN compared with patients with diabetes mellitus. In addition, the levels of uridine and dU did not alter in that previous study. It is well known that $\mathrm{dC}$ is deaminated to $\mathrm{dU}$ by the enzymes cytidine deaminase (CDA; EC 3.5.4.5) and deoxycytidine deaminase (DCD; EC 3.5.4.14) (66). Therefore, the increase in $\mathrm{dC}$ and the decrease in $\mathrm{dU}$ in MGN may be due to a decrease in the activity or expression of CDA and/or DCD. Notably, CDA activity may be affected by lymphomagenesis (67), and the development of cancer (68). However, alterations in the activity and/or expression of CDA in MGN require investigation in future studies to better understand the role that it serves in the pathogenesis of MGN.

There are no specific experimental data on the role of tryptamine in the pathogenesis of CKD. The present results demonstrated that patients with MGN had low urinary levels of tryptamine compared with the healthy controls. Arakaki et al (69) performed a fully automated computational metabolomics method to predict alterations in the metabolite levels in Jurkat T leukemia cells compared with normal cells. It was predicted that the levels of tryptamine may be lower in the cancer cells compared with the normal cells, as tryptamine exhibits antiproliferative activity. Tryptamine is an effective inhibitor of HeLa cell growth through the competitive inhibition of tryptophanyl-tRNA synthetase and subsequently, the inhibition of protein biosynthesis (69). Therefore, the decreased expression levels of tryptamine in patients with MGN may affect protein synthesis and the thickening of the GBM in the glomeruli. The exact mechanism of action of tryptamine in the pathogenesis of the disease is unclear and merits consideration in future studies.

Phenylalanine has demonstrated different fold-changes in the various causes of CKD. Sui et al (21) performed a pilot study using ${ }^{1} \mathrm{H}-\mathrm{NMR}$ spectroscopy to determine the disease-specific metabolite biomarker profiles in three groups: Healthy controls; low-risk patients who had renal biopsy-confirmed grades I-II IgA.N; and high-risk patients with grades IV-V nephropathy. High levels of serum phenylalanine were observed in the low-risk and high-risk patients compared with the healthy controls, which suggested that phenylalanine may

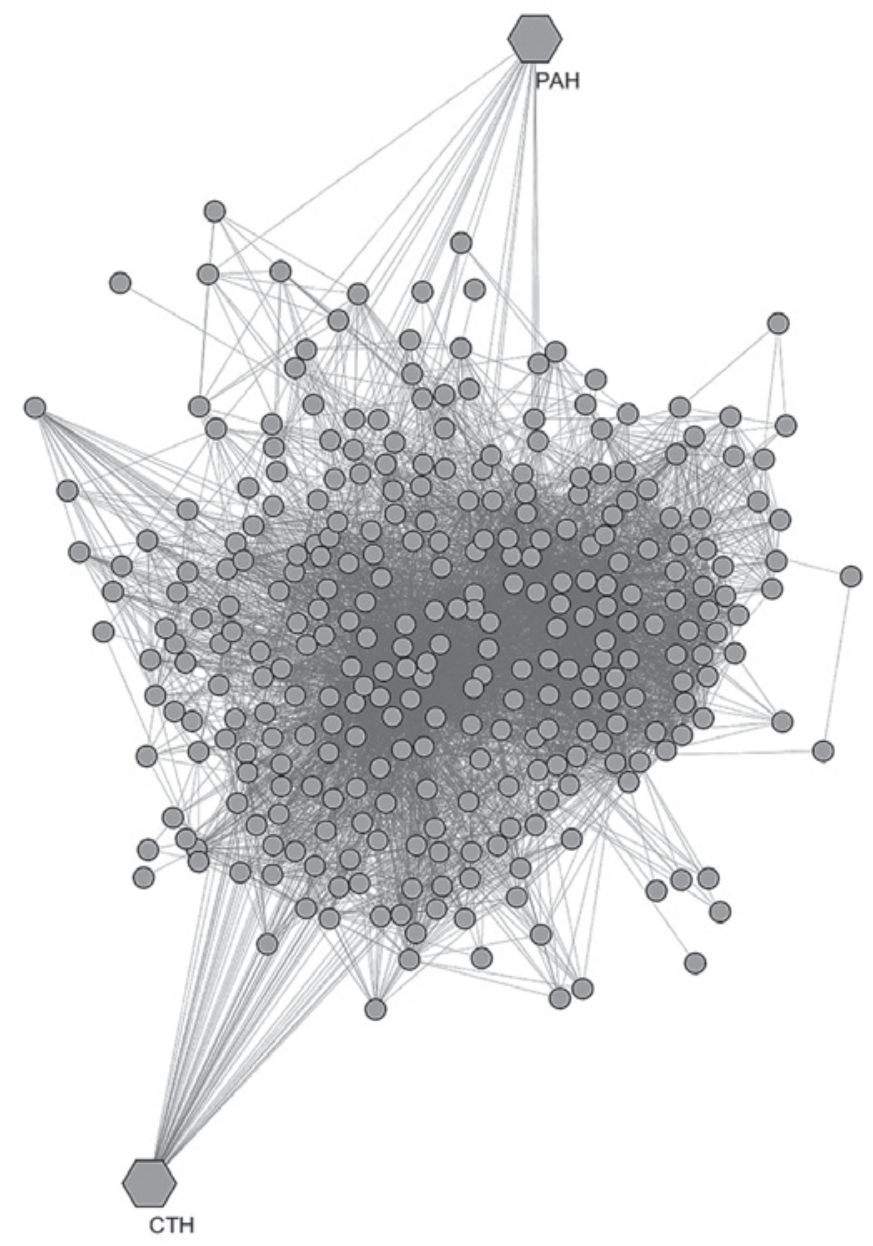

Figure 6. Protein-protein interaction network of MGN extracted from the Search Tool for the Retrieval of Interacting Genes database. The network was based on the proteins deduced from the component-reaction-enzyme-gene network associated with MGN. The hexagons represent $\mathrm{CTH}$ and PAH, which are seed nodes in cluster nos. 4 and 9, respectively. CTH, cystathionine $\gamma$-lyase; MGN, membranous glomerulonephritis; PAH, phenylalanine hydroxylase.

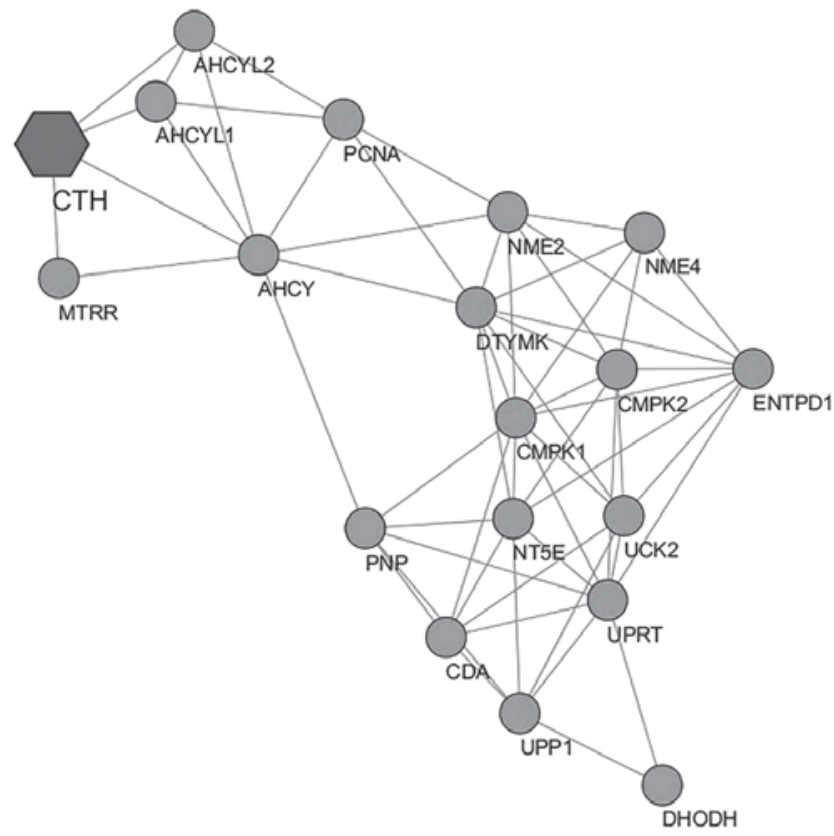

Figure 7. Cluster 4 includes CTH as a seed node. CTH, cystathionine $\gamma$-lyase. 
Table VI. Characteristics of 13 clusters in the protein-protein interaction network associated with membranous glomerulonephritis.

\begin{tabular}{|c|c|c|c|c|c|}
\hline Cluster no. & Score & No. of nodes & No. of edges & Seed & Degree \\
\hline 1 & 34.7 & 41 & 694 & DLAT & 9 \\
\hline 2 & 19.842 & 39 & 377 & IDH3G & 54 \\
\hline 3 & 10.926 & 55 & 295 & DHFRL1 & 30 \\
\hline $4^{\mathrm{a}}$ & 6.556 & 19 & 59 & CTH & 49 \\
\hline 5 & 4.8 & 16 & 36 & POLD1 & 33 \\
\hline 6 & 4.5 & 5 & 9 & GCLM & 23 \\
\hline 7 & 4.273 & 23 & 47 & IARS & 41 \\
\hline 8 & 4.0 & 6 & 10 & - & - \\
\hline $9^{a}$ & 3.0 & 3 & 3 & PAH & 17 \\
\hline 10 & 3.0 & 3 & 3 & NMNAT1 & 8 \\
\hline 11 & 3.0 & 3 & 3 & $\mathrm{DBH}$ & 9 \\
\hline 12 & 3.0 & 3 & 3 & UROC1 & 7 \\
\hline 13 & 3.0 & 3 & 3 & HIF1A & 14 \\
\hline
\end{tabular}

${ }^{a}$ Clusters 4 and 9 contain seed proteins which were involved in the component-reaction-enzyme-gene network.

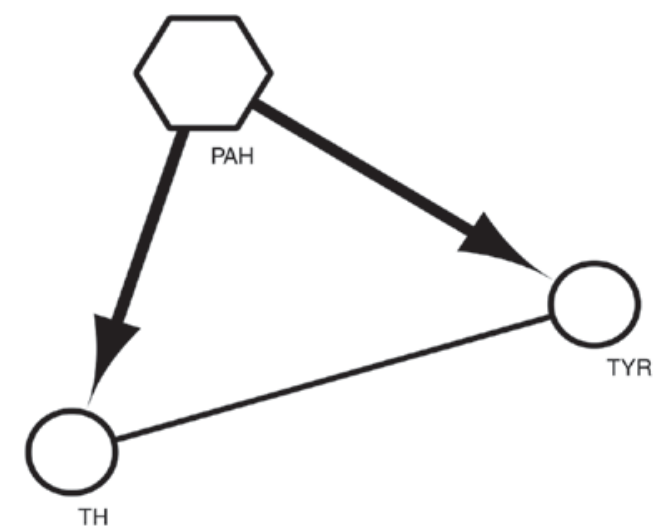

Figure 8. Cluster 9 includes PAH as a seed node. PAH, phenylalanine hydroxylase; TH, tyrosine hydroxylase; TYR, tyrosine.

be a potential biomarker for IgA.N. Li et al (70) reported high levels of serum phenylalanine in patients with renal function impairment; it was suggested that serum amino acid levels may be used for the early detection of CKD. Contradictory to these results, Li et al (71) detected low levels of serum phenylalanine in patients with lupus nephritis and idiopathic nephrotic syndrome using ultra-high-performance liquid chromatography coupled with high-resolution mass spectrometry. Since the role of phenylalanine in disease has rarely been reported, further investigation is warranted to elucidate the exact underlying mechanism.

According to the present results, fumarate was increased in the urine of patients with MGN. You et al (72) reported that the upregulation of podocyte NADPH oxidase (NOX)4 is sufficient to cause all of the glomerular features of diabetic kidney disease (DKD) in mice, including glomerular hypertrophy, mesangial matrix accumulation, podocyte dropout and GBM thickening. In addition, the authors demonstrated that pharmacological intervention with a NOX1/NOX4 inhibitor is able to attenuate the principal signs of DKD. NOX4 inhibition regulates fumarate levels, which led to the discovery that the fumarate hydratase enzyme, which is involved in the tricarboxylic acid cycle, is a downstream target of NOX4. You et al (72) identified fumarate to be a disease-promoting metabolite that stimulates endoplasmic reticulum (ER) stress, matrix gene expression, and hypoxia-inducible factor- $1 \alpha$ and TGF- $\beta$ production in mice with DKD. According to the results of the study by You et al (72), it may be hypothesized that fumarate is effective in causing glomeruli damage in MGN, including GBM thickening, by imposing ER stress, and thus has important effects on renal tissue.

In the present study, dopamine was significantly increased in the urine of patients with MGN. Pestana et al (73) reported that the urinary output of dopamine was lower in patients with salt-sensitive (SS) IgA.N compared with salt-resistant patients. Patients with SS IgA.N additionally exhibited lower creatinine clearance values and higher urinary protein excretion compared with salt-resistant patients. The authors speculated that the decreased renal dopamine synthesis in patients with SS IgA.N results from acquired tubulointerstitial injury. In addition, Zhang et al (74) reported that the decreased renal dopamine production may have important consequences in the underlying pathogenesis of DN. Notably, the hemodynamic actions of dopamine are dose-dependent; in low renal doses ( 0.5 to $2.5 \mu \mathrm{g} / \mathrm{kg} / \mathrm{min})$, dopamine stimulates the dopaminergic receptors in the renal and mesenteric vasculature, increases renal plasma flow, eGFR and sodium excretion in patients with normal renal function and with congestive heart failure (75). In addition, the decreased renal blood flow from the vasoconstriction caused by dopamine has been suggested as a contributing factor to the development of contrast-induced nephropathy; thus, dopamine failed to demonstrate a protective effect on renal function in patients undergoing contrast media exposure and was associated with a deleterious effect on the severity of renal failure and its duration (76). Therefore, it may be suggested that the 
Table VII. A list of thirteen potential biomarkers for membranous glomerulonephritis with high centralities in the componentreaction-enzyme-gene network and $1.5 \leq$ fold change $\leq 0.66$ compared with normal controls.

A, Upregulated

\begin{tabular}{|c|c|c|c|c|}
\hline Name & KEGG ID & Betweenness UnDir & Centroid UnDir & Fold change \\
\hline Dopamine & $\mathrm{C} 03758$ & $78,329.19$ & -938.0 & 1.65 \\
\hline Carnosine & C00386 & $33,611.40$ & -922.0 & 1.66 \\
\hline Fumarate & $\mathrm{C} 00122$ & $55,144.33$ & -822.0 & 1.55 \\
\hline Nicotinamide D-ribonucleotide & $\mathrm{C} 00455$ & $48,677.56$ & -732.0 & 2.01 \\
\hline AMP & $\mathrm{C} 00020$ & $235,243.60$ & -688.0 & 2.04 \\
\hline Pyridoxal & $\mathrm{C} 00250$ & $60,516.67$ & -1081.0 & 2.21 \\
\hline dGTP & $\mathrm{C} 00286$ & $78,288.00$ & -1066.0 & 1.84 \\
\hline
\end{tabular}

B, Downregulated

\begin{tabular}{|c|c|c|c|c|}
\hline Name & KEGG ID & Betweenness UnDir & Centroid UnDir & Fold change \\
\hline L-citrulline & $\mathrm{C} 00327$ & $19,650.12$ & -898.0 & 0.64 \\
\hline Nicotinamide & $\mathrm{C} 00153$ & $61,173.32$ & -638.0 & 0.36 \\
\hline L-phenylalanine & C00079 & $275,696.59$ & -416.0 & 0.55 \\
\hline Deoxyuridine & $\mathrm{C} 00526$ & $17,743.39$ & -1054.0 & 0.38 \\
\hline Tryptamine & C00398 & $14,025.28$ & -1052.0 & 0.57 \\
\hline Succinate & $\mathrm{C} 00042$ & $55,525.60$ & -1034.0 & 0.61 \\
\hline
\end{tabular}

KEGG, Kyoto Encyclopedia of Genes and Genomes; AMP, adenosine monophosphate; GTP, guanine triphosphate.

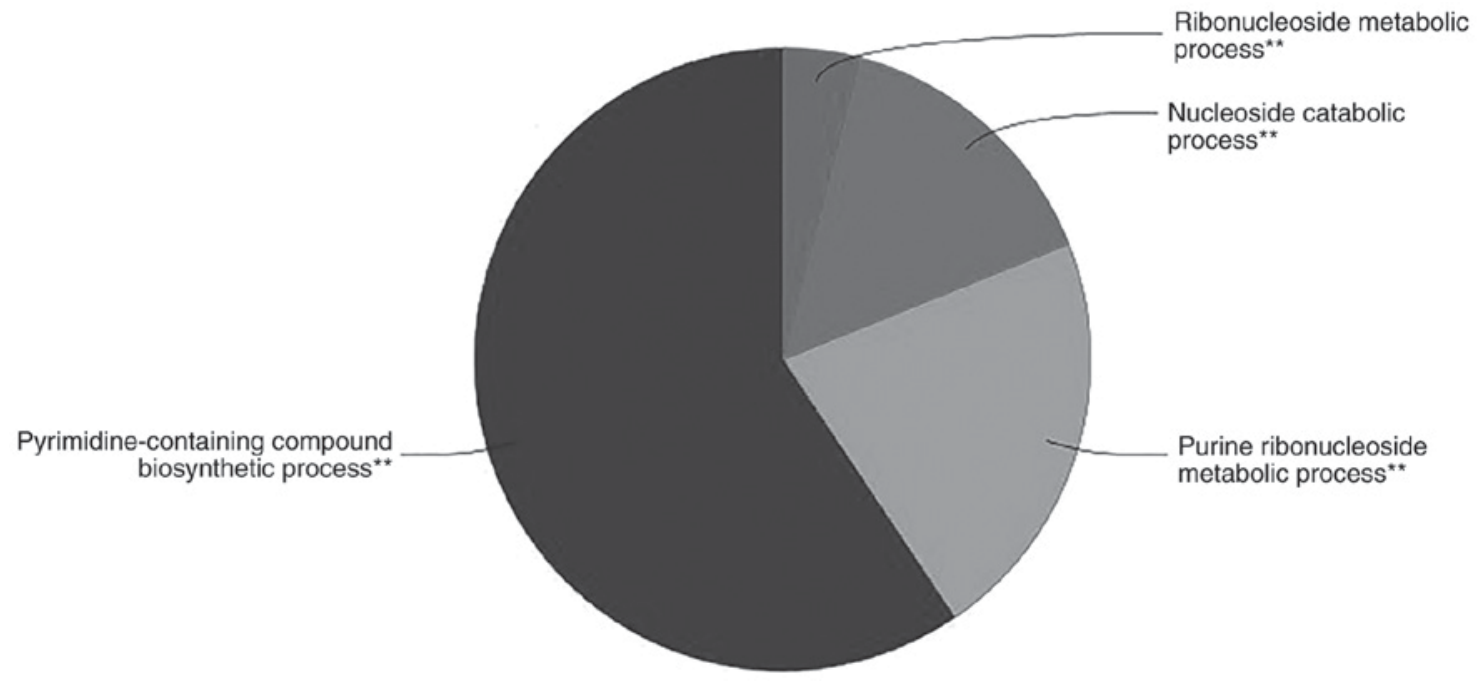

Figure 9. Gene ontology of the proteins involved in cluster 4 indicates that the protein family of cystathionine $\gamma$-lyase is involved in the 'pyrimidine-containing compound biosynthetic process', 'ribonucleoside metabolic process', 'nucleoside catabolic process', and 'purine ribonucleoside metabolic process'. "** $\mathrm{P}<0.01$.

renoprotective effects of dopamine occur only in low renal doses; conversely, decreased or increased levels of dopamine may have harmful effects on the kidney, which may result in enhanced nephropathy. In the present study, three derivatives of phenylalanine (33) were demonstrated to be increased in MGN: Tyrosine, tyramine and dopamine (Table II). Notably, the phenylalanine level decreased, which may be due to the alteration in the activity or expression of phenylalanine-4-hydroxylase $(\mathrm{PH} 4 \mathrm{H})$ that catalyzes the hydroxylation of the aromatic side-chain of phenylalanine to generate tyrosine (77). However, further research is required to validate this suggestion. In addition, one of the enriched BPs in the present dataset was 'aromatic amino acid family metabolic process'. Recently, Kalantari et al (62) reported that certain metabolic pathways were enriched in patients with severe IgA.N in comparison with patients with mild stages of the disease, and appeared to be associated with 'aromatic amino acid family metabolic process', including 'phenylalanine 


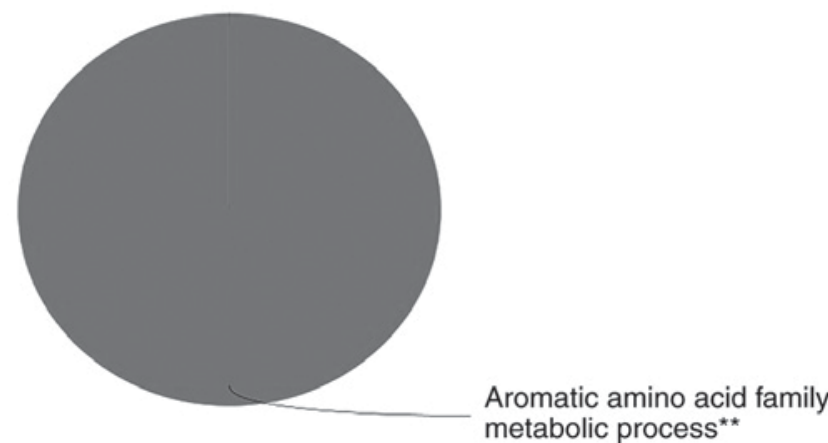

Figure 10. Cluster 9 gene ontology analysis by ClueGO demonstrates that the 'aromatic amino acid family metabolic process' is affected in patients with membranous glomerulonephritis. ${ }^{* *} \mathrm{P}<0.01$.

metabolism', 'tyrosine metabolism' and 'phenylalanine, tyrosine, and tryptophan biosynthesis'. The gene $P A H$, which was demonstrated to be a seed node for cluster 9, encodes the protein $\mathrm{PH} 4 \mathrm{H}$ (77).

According to the results of the present study, 'pyrimidine-containing compound biosynthetic process' is affected in MGN. 'Pyrimidine-containing compound biosynthetic process' refers to any BP that generates a pyrimidine-containing compound, including any nucleobase, nucleoside, nucleotide or nucleic acid that contains a pyrimidine base, from the derivatives without de novo synthesis (78). The thymidine kinase $(T k)$ gene codes for a cytosolic protein involved in the pyrimidine nucleotide salvage pathway. Dobrovolsky et al (79) created Tk KO mice to determine the biological significance of the $T k$ gene; it was demonstrated that all $T k \mathrm{KO}$ mice developed sclerosis of the kidney glomeruli and kidney failure. It was speculated that the pyrimidine nucleotide salvage pathway may be affected in $T k \mathrm{KO}$ mice in vivo. Therefore, it may be suggested that some of the glomeruli damage in MGN may be due to alterations in the levels of various pyrimidine-containing compounds, including the increased level of $\mathrm{dC}$ and the decreased level of $\mathrm{dU}$. However, the exact mechanism requires investigation in future studies.

According to the present study, "purine ribonucleoside metabolic process' was demonstrated to be affected in MGN patients. 'Purine ribonucleoside metabolic process' refers to the chemical reactions and pathways that involve any ribonucleoside, which is a nucleoside that has a purine base linked to a ribose (beta-D-ribofuranose) molecule (80). Recently, Mazumder et al (81) used a molecular modeling approach to demonstrate that purine nucleotides and/or their metabolites, including adenine, xanthine, hypoxanthine, 2,8-dihydroxyadenine and uric acid, are able to inhibit the activity of acetylcholinesterase (AChE) in the brain by interacting with the active site of the enzyme, which causes cognitive impairment (CI) in CKD patients. Notably, dementia and CI has been reported in 30-70\% of patients with CKD undergoing dialysis $(81,82)$. Therefore, Mazumder et al (81) suggested that a disturbed purine nucleotide metabolism may be a risk factor for CI in patients with CKD. High urinary levels of dGTP, AMP and adenosine in patients with MGN were detected; however, urinary levels of xanthine were low. The decreased level of xanthine in the urine of patients with MGN may be due to the impaired renal excretion of the molecule; therefore, the serum level of xanthine requires consideration in future studies. In addition, the binding affinity of other purine-associated metabolites, including AMP, adenosine and dGTP, to AChE require examination in future studies using experimental or computational methods. It may be suggested that 'purine ribonucleoside metabolic process' is affected in patients with MGN, which may result in the development of $\mathrm{CI}$ in patients with end-stage disease. It is noteworthy that the seed node for cluster 4 was the $C T H$ gene. The $C T H$ gene encodes the protein cystathionine $\gamma$-lyase (CGL) (83). The regulation of CGL in patients with MGN has not yet been verified. However, according to the results of the statistical modeling and network analysis, it may be suggested that the expression of CGL is affected in MGN, although further experimental gene expression analysis is required in order to verify this hypothesis.

The present study had certain limitations. Only Iranian patients were included in the present study and therefore, these findings may not entirely translate to patients of other ethnicities. However, the results may be useful for patient-specific treatments worldwide. Due to the fact that metabolomics analysis results in high-dimensional data, in which the generated data contain numerous variables and an insufficient number of samples, robust multivariate statistical analyses, including PLS-DA and OPLS-DA, are required to generate accurate results $(84,85)$. However, NMR and GC-MS studies consisting of a greater number of patients and healthy volunteers may enhance the statistical power and possibly reveal more significant metabolites that are associated with MGN. In addition, the same group of samples was not used in the NMR and GC-MS techniques of the study; therefore, the results may not be reliable and reproducible.

In conclusion, the present study successfully identified 13 differential metabolites considered to be potential biomarkers for MGN, according to their centralities and fold-changes. In addition, PPI analysis revealed two clusters with seed nodes $(C T H$ and $P A H)$, which were involved in the CREG network and were associated with MGN. The GO analysis additionally demonstrated five BP, which were significantly affected in MGN. According to the results of the present study, ${ }^{1} \mathrm{H}$ NMR and GC-MS techniques are able to monitor urinary metabolite profiles in patients with MGN and healthy individuals. OPLS-DA and PLS-DA are also two robust statistical methods, which are able to significantly identify differential metabolites derived from GC-MS and ${ }^{1} \mathrm{H}$ NMR data, respectively. Furthermore, Cytoscape and its associated plugins (MetScape, CentiScape, MCODE and ClueGO) proved to be powerful bioinformatics tools for identifying the most important metabolites in the CREG network, and for revealing clusters and BPs associated with MGN. The results may be used for novel research into diagnostic biomarkers, and may be useful for understanding the mechanisms underlying MGN.

\section{Acknowledgements}

The authors would like to thank Dr Salman Taheri who provided guidance. 


\section{Funding}

The present study was supported by a grant (grant no. 462.26) from the Chronic Kidney Disease Research Center, Shahid Beheshti University of Medical Sciences, Tehran, Iran.

\section{Availability of data and materials}

The datasets used and/or analyzed during the current study are available from the corresponding author on reasonable request.

\section{Authors' contributions}

AT, SK, AAO and MT designed the study. NMR and GC-MS experiments were conducted by AT, SK, AAO and KT. The statistical analysis (OPLS-DA and PLS-DA) was conducted by AT and SK. The network analysis was conducted and interpreted by AT and MT, and the results were analyzed and discussed by AT and MN. AT wrote the manuscript. All authors read and approved the final version of the manuscript.

\section{Ethics approval and consent to participate}

The present study was approved by the Ethics Committee of Shahid Beheshti University of Medical Sciences, Tehran, Iran. Informed consent was obtained from all participants.

\section{Patient consent for publication}

Not applicable.

\section{Competing interests}

The authors declare that they have no competing interests.

\section{References}

1. Cattran DC: Idiopathic membranous glomerulonephritis. Kidney Int 59: 1983-1994, 2001.

2. Kubota K, Hoshino J, Ueno T, Mise K, Hazue R, Sekine A, Yabuuchi J, Yamanouchi M, Suwabe T, Kikuchi K, et al: Phospholipase A2 Receptor-positive idiopathic membranous glomerulonephritis with onset at 95 years: Case report. Case Rep Nephrol Dial 6: 76-82, 2016.

3. Rivera F, Lopez-Gomez JM and Perez-Garcia R; Spanish Registry of Glomerulonephritis: Clinicopathologic correlations of renal pathology in Spain. Kidney Int 66: 898-904, 2004.

4. Santos FR: Membranous glomerulonephritis: New insights in pathophysiology and therapeutic approach. J Bras Nefrol 36: 59-62, 2014 (In Portuguese).

5. Huang YM, Zhou HR, Zhang L, Yang KK, Luo JX and Zhao HL: Spontaneous remission of membranous glomerulonephritis with successful fetal outcome: A case report and literature review. Medicine (Baltimore) 95: e4022, 2016.

6. Bomback AS and Fervenza FC: Membranous nephropathy: Approaches to treatment. Am J Nephrol 47 (Suppl 1): S30-S42, 2018.

7. Bertelli R, Bonanni A, Caridi G, Canepa A and Ghiggeri GM: Molecular and cellular mechanisms for proteinuria in minimal change disease. Fron Med (Lausanne) 5: 170, 2018.

8. Shimada S, Nakamichi T, Yamada G, Narumi K, Usubuchi H, Yamamoto T, Ichikawa S, Fukuhara N, Miyazaki M, Harigae $\mathrm{H}$, et al: Concurrent isolated IgG2-positive membranous nephropathy and malignant B-cell lymphoma. CEN Case Rep: 15 May, 2018 (Epub ahead of print).
9. Liu J and Wang W: Genetic basis of adult-onset nephrotic syndrome and focal segmental glomerulosclerosis. Front Med 11: 333-339, 2017.

10. Passos EM, Legallicier B and Godin M: Membranous nephropathy. Rev Prat 53: 2033-2038, 2003 (In French).

11. Tang KT, Tseng $\mathrm{CH}$, Hsieh TY and Chen DY: Induction therapy for membranous lupus nephritis: A systematic review and network meta-analysis. Int J Rheum Dis 21: 1163-1172, 2018.

12. Peng L, Wei SY, Li LT, He YX and Li B: Comparison of different therapies in high-risk patients with idiopathic membranous nephropathy. J Formos Med Assoc 115: 11-18, 2016.

13. Kraut JA and Madias NE: Adverse effects of the metabolic acidosis of chronic kidney disease. Adv Chronic Kidney Dis 24: 289-297, 2017.

14. Lai WL, Yeh TH, Chen PM, Chan CK, Chiang WC, Chen YM, Wu KD and Tsai TJ: Membranous nephropathy: A review on the pathogenesis, diagnosis, and treatment. J Formos Med Assoc 114: 102-111, 2015.

15. Abedi M and Gheisari Y: Nodes with high centrality in protein interaction networks are responsible for driving signaling pathways in diabetic nephropathy. PeerJ 3: e1284, 2015.

16. Debnath M, Prasad GB and Bisen PS: Omics technology. In: molecular diagnostics: Promises and Possibilities Springer, pp11-31, 2010.

17. Nagana Gowda GA and Raftery D: Can NMR solve some significant challenges in metabolomics? J Magn Reson 260: 144-160, 2015.

18. Markley JL, Brüschweiler R, Edison AS, et al: The future of NMR-based metabolomics. Curr Opin Biotechnol 43: 34-40, 2017

19. Dettmer K, Aronov PA and Hammock BD: Mass spectrometry-based metabolomics. Mass Spectrom Rev 26: 51-78, 2007.

20. Bingol K and Brüschweiler R: Two elephants in the room: New hybrid nuclear magnetic resonance and mass spectrometry approaches for metabolomics. Curr Opin Clin Nutr Metab Care 18: 471-477, 2015.

21. Sui W, Li L, Che W, Guimai Z, Chen J, Li W and Dai Y: A proton nuclear magnetic resonance-based metabonomics study of metabolic profiling in immunoglobulin a nephropathy. Clinics (Sao Paulo) 67: 363-373, 2012.

22. Ahmed KA and Chinnaiyan P: Applying metabolomics to understand the aggressive phenotype and identify novel therapeutic targets in glioblastoma. Metabolites 4: 740-750, 2014.

23. Nobakht BF, Aliannejad R, Rezaei-Tavirani M, Arefi Oskouie A, Naseri MT, Parastar H, Aliakbarzadeh G, Fathi F and Taheri S: NMR-and GC/MS-based metabolomics of sulfur mustard exposed individuals: A pilot study. Biomarkers 21: 479-489, 2016.

24. Zhang GQ and Hirasaki GJ: CPMG relaxation by diffusion with constant magnetic field gradient in a restricted geometry: Numerical simulation and application. J Magn Reson 163: 81-91, 2003.

25. Brown FF, Campbell ID, Kuchel PW and Rabenstein DC: Human erythrocyte metabolism studies by $1 \mathrm{H}$ spin echo NMR. FEBS Lett 82: 12-16, 1977.

26. Viant MR: Improved methods for the acquisition and interpretation of NMR metabolomic data. Biochem Biophys Res Commun 310: 943-948, 2003.

27. Holmes E, Foxall PJ, Nicholson JK, Neild GH, Brown SM, Beddell CR, Sweatman BC, Rahr E, Lindon JC, Spraul M, et al: Automatic data reduction and pattern recognition methods for analysis of $1 \mathrm{H}$ nuclear magnetic resonance spectra of human urine from normal and pathological states. Anal Biochem 220: 284-296, 1994.

28. Gavaghan CL, Holmes E, Lenz E, Wilson ID and Nicholson JK: An NMR-based metabonomic approach to investigate the biochemical consequences of genetic strain differences: Application to the C57BL10J and Alpk: ApfCD mouse. FEBS Lett 484: 169-174, 2000.

29. Holmes E, Nicholson JK, Nicholls AW, Lindon JC, Connor SC, Polley S and Connelly J: The identification of novel biomarkers of renal toxicity using automatic data reduction techniques and PCA of proton NMR spectra of urine. Chemometrics Intelligent Lab Systems 44: 245-255, 1998.

30. Gao J, Tarcea VG, Karnovsky A, Mirel BR, Weymouth TE, Beecher CW, Cavalcoli JD, Athey BD, Omenn GS, Burant CF and Jagadish HV: Metscape: A Cytoscape plug-in for visualizing and interpreting metabolomic data in the context of human metabolic networks. Bioinformatics 26: 971-973, 2010. 
31. Ma H, Sorokin A, Mazein A, Selkov A, Selkov E, Demin O and Goryanin I: The Edinburgh human metabolic network reconstruction and its functional analysis. Mol Syst Biol 3: 135, 2007.

32. Wishart DS, Tzur D, Knox C, Eisner R, Guo AC, Young N Cheng D, Jewell K, Arndt D, Sawhney S, et al: HMDB: The human metabolome database. Nucleic Acids Res 35 (Database Issue): D521-D526, 2007.

33. Kanehisa M and Goto S: KEGG: Kyoto encyclopedia of genes and genomes. Nucleic Acids Res 28: 27-30, 2000.

34. Karnovsky A, Weymouth T, Hull T, Tarcea VG, Scardoni G, Laudanna C, Sartor MA, Stringer KA, Jagadish HV, Burant C, et al: Metscape 2 bioinformatics tool for the analysis and visualization of metabolomics and gene expression data. Bioinformatics 28: 373-380, 2011

35. Hao T, Peng W, Wang Q, Wang B and Sun J: Reconstruction and application of protein-protein interaction network. Int J Mol Sci 17: pii: E907, 2016.

36. Zali $\mathrm{H}$ and Rezaei Tavirani M: Meningioma protein-protein interaction network. Arch Iran Med 17: 262-272, 2014

37. Szklarczyk D, Morris JH, Cook H, Kuhn M, Wyder S, Simonovic M, Santos A, Doncheva NT, Roth A, Bork P, et al: The STRING database in 2017: Quality-controlled protein-protein association networks, made broadly accessible. Nucleic Acids Res 45: D362-D368, 2017

38. Saito R, Smoot ME, Ono K, Ruscheinski J, Wang PL, Lotia S, Pico AR, Bader GD and Ideker T: A travel guide to Cytoscape plugins. Nature Methods 9: 1069-1076, 2012.

39. Bader GD and Hogue CW: An automated method for finding molecular complexes in large protein interaction networks. BMC Bioinformatics 4: 2, 2003

40. Bindea G, Mlecnik B, Hackl H, Charoentong P, Tosolini M Kirilovsky A, Fridman WH, Pagès F, Trajanoski Z and Galon J: ClueGO: A Cytoscape plug-in to decipher functionally grouped gene ontology and pathway annotation networks. Bioinformatics 25: 1091-1093, 2009.

41. Hallan S, Afkarian M, Zelnick LR, Kestenbaum B, Sharma S, Saito R, Darshi M, Barding G, Raftery D, Ju W, et al: Metabolomics and gene expression analysis reveal down-regulation of the citric acid (TCA) cycle in non-diabetic CKD patients EBioMedicine 26: 68-77, 2017.

42. Toyohara T, Akiyama Y, Suzuki T, Takeuchi Y, Mishima E, Tanemoto M, Momose A, Toki N, Sato H, Nakayama M, et al: Metabolomic profiling of uremic solutes in CKD patients. Hypertens Res 33: 944-952, 2010.

43. Lin IC, Hsu CN, Lo MH, Chien SJ and Tain YL: Low urinary citrulline/arginine ratio associated with blood pressure abnormalities and arterial stiffness in childhood chronic kidney disease. J Am Soc Hypertens 10: 115-123, 2016.

44. Janssen B, Hohenadel D, Brinkkoetter P, Peters V, Rind N, Fischer C, Rychlik I, Cerna M, Romzova M, de Heer E, et al: Carnosine as a protective factor in diabetic nephropathy: Association with a leucine repeat of the carnosinase gene CNDP1. Diabetes 54: 2320-2327, 2005.

45. Albrecht T, Schilperoort M,Zhang S,Braun JD, Qiu J, Rodriguez A Pastene DO, Krämer BK, Köppel H, Baelde H, et al: Carnosine attenuates the development of both type 2 diabetes and diabetic nephropathy in BTBR ob/ob mice. Sci Rep 7: 44492, 2017.

46. Kaori H, Hiroyuki S, Takako H, Makoto S, Satsuki I, Tomoyoshi S and Hiroshi I: Use of serum and urine metabolome analysis for the detection of metabolic changes in patients with stage 1-2 chronic kidney disease. Nephrourol Monthly 2011: 164-171, 2011

47. Ulvik A, Midttun $\varnothing$, Pedersen ER, Eussen SJ, Nygård O and Ueland PM: Evidence for increased catabolism of vitamin B-6 during systemic inflammation. Am J Clin Nutr 100: 250-255, 2014.

48. Chen CH, Yeh EL, Chen CC, Huang SC and Huang YC: Vitamin B-6, independent of homocysteine, is a significant factor in relation to inflammatory responses for chronic kidney disease and hemodialysis patients. Biomed Res Int 2017: 7367831, 2017

49. Zhang P, Tsuchiya K, Kinoshita T, Kushiyama H, Suidasari S, Hatakeyama M, Imura H, Kato N and Suda T: Vitamin B6 prevents IL-1 $\beta$ protein production by inhibiting nlrp3 inflammasome activation. J Biol Chem 291: 24517-24527, 2016.

50. Egashira Y, Nagaki S and Sanada H: Tryptophan-niacin metabolism in rat with puromycin aminonucleoside-induced nephrosis. Int J Vitam Nutr Res 76: 28-33, 2006

51. Zhao X, Wu H, Guo B, Dong R, Qiu Y and Ma PX: Antibacterial anti-oxidant electroactive injectable hydrogel as self-healing wound dressing with hemostasis and adhesiveness for cutaneous wound healing. Biomaterials 122: 34-47, 2017.
52. Demaria M, Ohtani N, Youssef SA, Rodier F, Toussaint W, Mitchell JR, Laberge RM, Vijg J, Van Steeg H, Dollé ME, et al: An essential role for senescent cells in optimal wound healing through secretion of PDGF-AA. Dev Cell 31: 722-733, 2014.

53. Wang WJ, Cai GY and Chen XM: Cellular senescence, senescence-associated secretory phenotype, and chronic kidney disease. Oncotarget 8: 64520-64533, 2017.

54. Kume S, Haneda M, Kanasaki K, Sugimoto T, Araki S, Isshiki K, Isono M, Uzu T, Guarente L, Kashiwagi A and Koya D: SIRT1 inhibits transforming growth factor beta-induced apoptosis in glomerular mesangial cells via Smad7 deacetylation. J Biol Chem 282: 151-158, 2007

55. Kume S, Haneda M, Kanasaki K, Sugimoto T, Araki S, Isono M, Isshiki K, Uzu T, Kashiwagi A and Koya D: Silent information regulator 2 (SIRT1) attenuates oxidative stress-induced mesangial cell apoptosis via p53 deacetylation. Free Radical Biol Med 40: 2175-2182, 2006

56. Li C, Cai F, Yang Y, Zhao X, Wang C, Li J, Jia Y, Tang J and Liu Q: Tetrahydroxystilbene glucoside ameliorates diabetic nephropathy in rats: Involvement of SIRT1 and TGF- $\beta 1$ pathway. Eur J Pharmacol 649: 382-389, 2010.

57. Kalantari S, Nafar M, Samavat S, Parvin M, Nobakht M Gh BF and Barzi F: ${ }^{1} \mathrm{H}$ NMR-based metabolomics exploring urinary biomarkers correlated with proteinuria in focal segmental glomerulosclerosis: A pilot study. Magn Reson Chem: Jun 19, 2016 (Epub ahead of print).

58. Revollo JR, Grimm AA and Imai S: The regulation of nicotinamide adenine dinucleotide biosynthesis by Nampt/PBEF/visfatin in mammals. Curr Opin Gastroenterol 23: 164-170, 2007.

59. Chen Y, Liang Y, Hu T, Wei R, Cai C, Wang P, Wang L, Qiao W and Feng L: Endogenous Nampt upregulation is associated with diabetic nephropathy inflammatory-fibrosis through the NF- $\kappa B$ p65 and Sirt1 pathway; NMN alleviates diabetic nephropathy inflammatory-fibrosis by inhibiting endogenous Nampt. Exp Ther Med 14: 4181-4193, 2017.

60. Hasegawa K, Wakino S, Simic P, Sakamaki Y, Minakuchi H, Fujimura K, Hosoya K, Komatsu M, Kaneko Y, Kanda T, et al: Renal tubular Sirt1 attenuates diabetic albuminuria by epigenetically suppressing Claudin-1 overexpression in podocytes. Nat Med 19: 1496-1504, 2013

61. Helmering J, Juan T, Li CM, Chhoa M, Baron W, Gyuris T, Richards WG, Turk JR, Lawrence J, Cosgrove PA, et al: A mutation in Ampd2 is associated with nephrotic syndrome and hypercholesterolemia in mice. Lipids Health Dis 13: 167, 2014.

62. Kalantari S, Nafar M, Samavat S and Parvin M: 1 H NMR-based metabolomics study for identifying urinary biomarkers and perturbed metabolic pathways associated with severity of IgA nephropathy: A pilot study. Magn Reson Chem 55: 693-699, 2017.

63. Furman PA, Lambe CU and Nelson DJ: Effect of acyclovir on the deoxyribonucleoside triphosphate pool levels in vero cells infected with herpes simplex virus type 1. Am J Med 73: 14-17, 1982.

64. Gamboa JL, Billings FT VI, Bojanowski MT, Gilliam LA, Yu C, Roshanravan B, Roberts LJ II, Himmelfarb J, Ikizler TA and Brown NJ: Mitochondrial dysfunction and oxidative stress in patients with chronic kidney disease. Physiol Rep 4: pii: e12780, 2016.

65. Xia JF, Hu P, Liang QL, Zou TT, Wang YM and Luo GA: Correlations of creatine and six related pyrimidine metabolites and diabetic nephropathy in Chinese type 2 diabetic patients. Clin Biochem 43: 957-962, 2010.

66. Tyburski JB, Patterson AD, Krausz KW, Slavík J, Fornace AJ Jr, Gonzalez FJ and Idle JR: Radiation metabolomics. 2. Dose-and time-dependent urinary excretion of deaminated purines and pyrimidines after sublethal gamma-radiation exposure in mice. Radiat Res 172: 42-57, 2009.

67. Gu X, Shivarov V and Strout MP: The role of activation-induced cytidine deaminase in lymphomagenesis. Curr Opin Hematol 19: 292-298, 2012

68. Marusawa H, Takai A and Chiba T: Role of activation-induced cytidine deaminase in inflammation-associated cancer development. In: Advances in immunology Elsevier, pp109-141, 2011.

69. Arakaki AK, Mezencev R, Bowen NJ, Huang Y, McDonald JF and Skolnick J: Identification of metabolites with anticancer properties by computational metabolomics. Mol Cancer 7: 57, 2008.

70. Li R, Dai J and Kang H: The construction of a panel of serum amino acids for the identification of early chronic kidney disease patients. J Clin Lab Anal 32, 2018. 
71. Li J, Xie XW, Zhou H, Wang B, Zhang MJ and Tang FY: Metabolic profiling reveals new serum biomarkers of lupus nephritis. Lupus 26: 1166-1173, 2017.

72. You YH, Quach T, Saito R, Pham J and Sharma K: Metabolomics reveals a key role for fumarate in mediating the effects of NADPH oxidase 4 in diabetic kidney disease. J Am Soc Nephrol 27: 466-481, 2016.

73. Pestana M, Santos J, Santos A, Coroas A, Correia F, Serrão P, Valbuena C and Soares-da-Silva P: Renal dopamine and salt sensitivity of blood pressure in IgA nephropathy. Kidney Blood Press Res 27: 78-87, 2004.

74. Zhang MZ, Yao B, Yang S, Yang H, Wang S, Fan X, Yin H, Fogo AB, Moeckel GW and Harris RC: Intrarenal dopamine inhibits progression of diabetic nephropathy. Diabetes 61: 2575-2584, 2012.

75. Gare M, Haviv YS, Ben-Yehuda A, Rubinger D, Bdolah-Abram T, Fuchs S, Gat O, Popovtzer MM, Gotsman MS and Mosseri M: The renal effect of low-dose dopamine in high-risk patients undergoing coronary angiography. J Am Coll Cardiol 34: 1682-1688, 1999.

76. Sudarsky D and Nikolsky E: Contrast-induced nephropathy in interventional cardiology. Int J Nephrol Renovasc Dis 4: 85-99, 2011.

77. Fitzpatrick PF: Tetrahydropterin-dependent amino acid hydroxylases. Annu Rev Biochem 68: 355-381, 1999.

78. Cherry JM, Hong EL, Amundsen C, Balakrishnan R, Binkley G, Chan ET, Christie KR, Costanzo MC, Dwight SS, Engel SR, et al: Saccharomyces Genome database: The genomics resource of budding yeast. Nucleic Acids Res 40 (Database Issue): D700-D705, 2012
79. Dobrovolsky VN, Bucci T, Heflich RH, Desjardins J and Richardson FC: Mice deficient for cytosolic thymidine kinase gene develop fatal kidney disease. Mol Genet Metab 78: 1-10, 2003.

80. Smith CL, Blake JA, Kadin JA, Richardson JE and Bult CJ; Mouse Genome Database Group: Mouse genome database (MGD)-2018: Knowledgebase for the laboratory mouse. Nucleic Acids Res 46: D836-D842, 2017.

81. Mazumder MK, Phukan BC, Bhattacharjee A and Borah A: Disturbed purine nucleotide metabolism in chronic kidney disease is a risk factor for cognitive impairment. Med Hypotheses 111: 36-39, 2018.

82. Sarnak MJ, Tighiouart H, Scott TM, Lou KV, Sorensen EP, Giang LM, Drew DA, Shaffi K, Strom JA, Singh AK and Weiner DE: Frequency of and risk factors for poor cognitive performance in hemodialysis patients. Neurology 80: 471-480, 2013.

83. Apweiler R, Bairoch A, Wu CH, Barker WC, Boeckmann B, Ferro S, Gasteiger E, Huang H, Lopez R, Magrane M, et al: UniProt: The universal protein knowledgebase. Nucleic Acids Res 32 (Database Issue): D115-D119, 2004.

84. Gromski PS, Muhamadali H, Ellis DI, Xu Y, Correa E, Turner ML and Goodacre R: A tutorial review: Metabolomics and partial least squares-discriminant analysis-a marriage of convenience or a shotgun wedding. Anal Chim Acta 879: 10-23, 2015.

85. Liland KH: Multivariate methods in metabolomics-from pre-processing to dimension reduction and statistical analysis. TrAC Trends Analytical Chemistry 30: 827-841, 2011. 